\title{
REMARKS ON DRIFT ESTIMATION FOR DIFFUSION PROCESSES*
}

\author{
YVO POKERN ${ }^{\dagger}$, ANDREW M. STUART ${ }^{\ddagger}$, AND ERIC VANDEN-EIJNDEN $\$$
}

\begin{abstract}
In applications such as molecular dynamics it is of interest to fit Smoluchowski and Langevin equations to data. Practitioners often achieve this by a variety of seemingly ad hoc procedures such as fitting to the empirical measure generated by the data and fitting to properties of autocorrelation functions. Statisticians, on the other hand, often use estimation procedures, which fit diffusion processes to data by applying the maximum likelihood principle to the path-space density of the desired model equations, and through knowledge of the properties of quadratic variation. In this paper we show that the procedures used by practitioners and statisticians to fit drift functions are, in fact, closely related and can be thought of as two alternative ways to regularize the (singular) likelihood function for the drift. We also present the results of numerical experiments which probe the relative efficacy of the two approaches to model identification and compare them with other methods such as the minimum distance estimator.
\end{abstract}

Key words. parameter estimation, diffusion process, nonparametric estimation, maximum likelihood principle, minimum distance estimator, reversible diffusion process, molecular dynamics, Langevin equation

AMS subject classifications. 62M05, 65C 30

DOI. $10.1137 / 070694806$

1. Introduction. In many applications (such as molecular dynamics, econometrics, atmospheric sciences, and signal processing) it is of interest to fit a diffusion process to a time-series. The data may come from experiments, or from the numerical simulation of larger and more complex models, either deterministic or stochastic. The objective of the present paper is to discuss some issues that arise when applying a maximum likelihood inference method to this problem. In so doing, we will highlight some connections between this approach, favored by statisticians, and other approaches used in the physics and chemistry literature.

To introduce the maximum likelihood inference framework and some of the issues that we will discuss, it is useful to consider first the specific case when it is known that the data is consistent with an Itô stochastic differential equation of the form

$$
\dot{X}_{t}=-\nabla V_{0}\left(X_{t}\right)+\sqrt{2 \beta^{-1}} \dot{W}_{t} .
$$

This equation is often referred to as the Smoluchowski or overdamped Langevin equation in the chemical-physics literature. Precise statements of the observations about this problem that we make in this introductory section will be provided in section 2.1 . In section 2.2 we consider general reversible diffusions and in section 3 the (nonreversible) second order Langevin equation.

In (1.1), $W_{t}$ is a standard $d$-dimensional Brownian motion in $\mathbb{R}^{d}, \beta>0$ is a constant playing the role of the inverse temperature, and $V_{0}: \mathbb{R}^{d} \rightarrow \mathbb{R}$ is a potential, which we assume $C^{2}$, bounded from below and with a growth condition at infinity to

*Received by the editors June 19, 2007; accepted for publication (in revised form) April 20, 2009; published electronically October 22, 2009.

http://www.siam.org/journals/mms/8-1/69480.html

${ }^{\dagger}$ Department of Statistics, University of Warwick, Coventry CV4 7AL, UK (Y.Pokern@warwick. ac.uk).

${ }^{\ddagger}$ Mathematics Institute, University of Warwick, Coventry CV4 7AL, UK (A.M.Stuart@warwick. ac.uk).

$\S$ Courant Institute, New York University, New York, NY 10012 (eve2@cims.nyu.edu). 
guarantee that $e^{-\beta V_{0}}$ is integrable. In this case, the process defined by (1.1) is ergodic with respect to the Boltzmann-Gibbs measure associated with $V_{0}$ whose density is

$$
\rho_{0}(x)=Z^{-1} e^{-\beta V_{0}(x)}, \quad \text { where } \quad Z=\int_{\mathbb{R}^{d}} e^{-\beta V_{0}(x)} d x .
$$

We assume that $\beta$ is known and that we wish to estimate the potential $V_{0}$ from the data, i.e., from a sample path $\left\{X_{t}\right\}_{t \in[0, T]}$ for some $T>0$. For the time being we assume that a continuous sample of the path is available; later on in the paper, we will also discuss the problem when $X_{t}$ is sampled at discrete times. To see how the problem of estimating $V_{0}$ given $\beta$ can be cast into a maximum likelihood inference problem, let $Z_{t}$ solve (1.1) for $V_{0} \equiv 0$ so that

$$
\dot{Z}_{t}=\sqrt{2 \beta^{-1}} \dot{W}_{t},
$$

and let $\mathbb{P}$ and $\mathbb{Q}$ be the path-space measures generated on $[0, T]$ by (1.1) and (1.3), respectively. Then these measures are absolutely continuous with Radon-Nikodym derivative

$$
\frac{d \mathbb{P}}{d \mathbb{Q}}=\exp \left(-T I_{T}(X)\right)
$$

where

$$
I_{T}(X)=\frac{\beta}{4 T} \int_{0}^{T}\left(\left|\nabla V_{0}\left(X_{t}\right)\right|^{2} d t+2\left\langle\nabla V_{0}\left(X_{t}\right), d X_{t}\right\rangle\right)
$$

the angle brackets $\langle\cdot, \cdot\rangle$ denote the Euclidean inner product on $\mathbb{R}^{d}$ and $|\cdot|$ the Euclidean norm, and the integral with respect to $d X_{t}$ is to be understood in the Itô sense. The functional $I_{T}(X)$ given by (1.5) is proportional to the negative logarithm of the probability density of the path $\left\{X_{t}\right\}_{t \in[0, T]}$ with respect to the measure on path-space generated by (1.3). When a single path $\left\{X_{t}\right\}_{t \in[0, T]}$ is given, if we evaluate (1.5) with potential $V$ rather than $V_{0}$, this object becomes a functional of $V$. This functional is the negative of the log likelihood function for $V$ :

$$
\mathcal{I}_{T}(V)=\frac{\beta}{4 T} \int_{0}^{T}\left(\left|\nabla V\left(X_{t}\right)\right|^{2} d t+2\left\langle\nabla V\left(X_{t}\right), d X_{t}\right\rangle\right) .
$$

Thus, it is natural to try to minimize (1.6) over $V$ to obtain the maximum likelihood estimator (MLE) for this function. Indeed, using (1.1), (1.6) can be written as

$$
\begin{aligned}
\mathcal{I}_{T}(V) & =\frac{\beta}{4 T} \int_{0}^{T}\left(\left|\nabla V\left(X_{t}\right)\right|^{2}-2\left\langle\nabla V\left(X_{t}\right), \nabla V_{0}\left(X_{t}\right)\right\rangle\right) d t \\
& +\frac{\sqrt{2 \beta}}{2 T} \int_{0}^{T}\left\langle\nabla V\left(X_{t}\right), d W_{t}\right\rangle .
\end{aligned}
$$

Letting $T \rightarrow \infty$, the stochastic integral in this expression tends to 0 almost surely (a.s.), whereas the time integral converges a.s. toward an expectation with respect to the equilibrium measure with density (1.2). In other words, as $T \rightarrow \infty, \mathcal{I}_{T}(V)$ converges a.s. to the functional $\mathcal{I}_{\infty}(V)$ given by

$$
\mathcal{I}_{\infty}(V)=\frac{\beta}{4} \int_{\mathbb{R}^{d}}\left(|\nabla V(x)|^{2}-2\left\langle\nabla V(x), \nabla V_{0}(x)\right\rangle\right) \rho_{0}(x) d x .
$$


This functional is quadratic and convex in $\nabla V$ and, by completing the square, it is clearly minimized when $\nabla V=\nabla V_{0}$, i.e., when $V=V_{0}+C$, where $C$ is an arbitrary (and irrelevant) constant. Thus the MLE for $-\nabla V_{0}$ given by maximizing the limiting functional (1.8) is indeed the actual drift in (1.1).

The problem, however, is that the data $\left\{X_{t}\right\}_{t \in[0, T]}$ is finite, $T<\infty$, i.e., we are obliged to work with (1.6) and have no access to its infinite time limit (1.8). To see what problems this creates, let us first put (1.6) in a more convenient form by converting the Itô stochastic integral $\left\langle\nabla V\left(X_{t}\right), d X_{t}\right\rangle$ into the Stratonovich integral using

$$
\left\langle\nabla V\left(X_{t}\right), \circ d X_{t}\right\rangle=\left\langle\nabla V\left(X_{t}\right), d X_{t}\right\rangle+\beta^{-1} \Delta V\left(X_{t}\right) d t .
$$

Since $\left\langle\nabla V\left(X_{t}\right)\right.$, od $\left.X_{t}\right\rangle=d V\left(X_{t}\right)$, this gives

$$
\mathcal{I}_{T}(V)=\frac{\beta}{2 T}\left(V\left(X_{T}\right)-V\left(X_{0}\right)\right)+\frac{\beta}{4 T} \int_{0}^{T}\left(\left|\nabla V\left(X_{t}\right)\right|^{2}-2 \beta^{-1} \Delta V\left(X_{t}\right)\right) d t .
$$

The time integral in (1.9) can be transformed into a configuration integral using the occupation measure $\mu_{T}$ defined such that, for any Borel set $B \subset \mathbb{R}^{d}$, one has

$$
\mu_{T}(B)=\frac{1}{T} \int_{0}^{T} \mathbf{1}_{B}\left(X_{t}\right) d t,
$$

where $\mathbf{1}_{B}(x)$ is the indicator function of the set $B$. The measure $\mu_{T}$ is the finite time equivalent of the equilibrium measure $\rho_{0}(x) d x$ entering (1.8). Using $\mu_{T}$, we can write (1.9) as

$$
\mathcal{I}_{T}(V)=\frac{\beta}{2 T}\left(V\left(X_{T}\right)-V\left(X_{0}\right)\right)+\frac{\beta}{4} \int_{\mathbb{R}^{d}}\left(|\nabla V(x)|^{2}-2 \beta^{-1} \Delta V(x)\right) \mu_{T}(d x) .
$$

This expression (1.11) makes it apparent why an attempt to directly minimize this functional over $V$ is a bad idea. When $d=1$, the occupation measure $\mu_{T}$ has the scaled local time $L_{T}^{x} / T$ of the process $\left\{X_{t}\right\}_{t \in[0, T]}$ as a density, but $L_{T}^{x}$ is only Hölder continuous up to $C^{0, \frac{1}{2}}(\mathbb{R})$. Indeed, $L_{T}^{x}$ has the fine-scale properties of a diffusion process (cf. the Ray-Knight description of Brownian local times [15]). In the appendix, we show that (1.11) evaluated with $\mu_{T}(d x)=w(x) d x$, where $w(x)$ is a one-dimensional Brownian motion, is not bounded from below. When $d>1$, the situation is even worse, as $\mu_{T}$ is singular with respect to the Lebesgue measure since it is supported on $\left\{X_{t}\right\}_{t \in[0, T]}$. Thus $\mathcal{I}_{T}(V)$ must be regularized in some way to become useful. There are at least three obvious ways to perform such a regularization.

1. The first way, which we will not discuss in this paper, is to adopt a Bayesian nonparametric approach in which a prior measure on $V$ is introduced that is supported only on sufficiently regular functions. By sampling from this measure and using the exponential of the negative of (1.6) or, equivalently, (1.11) as reweighting density, it is possible to sample the posterior distribution of $V$ given the data $\left\{X_{t}\right\}_{t \in[0, T]}$. This approach is discussed in [19], and we refer the reader to that paper for details.

2. A second way to regularize (1.11) is to assume a parametric form for $V$, e.g., as a linear combination of smooth basis functions $f_{i}(x)$,

$$
V(x, \theta)=\sum_{j=1}^{N} \theta_{i} f_{i}(x),
$$

Copyright (c) by SIAM. Unauthorized reproduction of this article is prohibited. 
where $\theta_{1}, \ldots, \theta_{N}$ are weights. By substituting (1.12) into (1.6), one is left with a quadratic function of $\theta=\left(\theta_{1}, \ldots, \theta_{N}\right) \in \mathbb{R}^{N}$,

$$
\mathcal{I}_{T}(\theta)=\frac{\beta}{4 T} \int_{0}^{T}\left(\left|\sum_{i=1}^{N} \theta_{i} \nabla f_{i}\left(X_{t}\right)\right|^{2} d t+2 \sum_{i=1}^{N} \theta_{i}\left\langle\nabla f_{i}\left(X_{t}\right), d X_{t}\right\rangle\right) .
$$

For an appropriate choice of $f_{i}(x)$, this quadratic function of $\theta$ is convex and therefore has a unique minimum $\hat{\theta}$ which can be found by solving a linear algebraic system. This approach is the one often adopted in the statistics literature to identify a parametric approximation to the MLE of $V$. We will refer to it as the parametric approach. Notice that for this approach to work it is crucial that the sum in (1.12) be finite, since it is this which regularizes the functional (1.6); the actual (nonparametric) MLE for $V$ will not exist in general.

3. A third way to regularize (1.11) is to regularize the measure $\mu_{T}(d x)$ and replace it by $\rho_{T}(x) d x$, where $\rho_{T}(x)>0$ is a smooth probability density function. With this substitution, (1.11) becomes

$$
\mathcal{I}_{T}(V)=\frac{\beta}{2 T}\left(V\left(X_{T}\right)-V\left(X_{0}\right)\right)+\tilde{\mathcal{I}}_{T}(V),
$$

where

$$
\tilde{\mathcal{I}}_{T}(V)=\frac{\beta}{4} \int_{\mathbb{R}^{d}}\left(|\nabla V(x)|^{2}-2 \beta^{-1} \Delta V(x)\right) \rho_{T}(x) d x .
$$

If $T$ is large enough, it is reasonable to neglect the first term on the right-hand side of (1.14), i.e., approximate $\mathcal{I}_{T}(V)$ by $\tilde{\mathcal{I}}_{T}(V)$. To identify the minimizer of $\tilde{\mathcal{I}}_{T}(V)$, note that if $\rho_{T}(x)>0$, and for potentials $V$ such that $\lim _{x \rightarrow \infty} \nabla V(x) \rho_{T}(x)=0$, an integration by parts yields

$$
\begin{aligned}
\tilde{\mathcal{I}}_{T}(V) & =\frac{\beta}{4} \int_{\mathbb{R}^{d}}\left(|\nabla V(x)|^{2}+2 \beta^{-1}\left\langle\nabla V(x), \nabla \log \rho_{T}(x)\right\rangle\right) \rho_{T}(x) d x \\
& =\frac{\beta}{4} \int_{\mathbb{R}^{d}}\left(\left|\nabla V(x)+\beta^{-1} \nabla \log \rho_{T}(x)\right|^{2}-\beta^{-2}\left|\nabla \log \rho_{T}(x)\right|^{2}\right) \rho_{T}(x) d x .
\end{aligned}
$$

This last expression shows that the minimizer of $\tilde{\mathcal{I}}_{T}(V)$ is unique up to a constant and given by

$$
\hat{V}(x)=-\beta^{-1} \log \rho_{T}(x)+C^{\prime},
$$

where $C^{\prime}$ is an arbitrary constant. This expression for $V$ is the one usually adopted in the physics and chemistry literature and we will refer to it as the nonparametric approach since (1.16) and, hence, (1.17) involve no direct parametrization of $V$. Notice, however, that this approach leaves as an auxiliary problem the issue of determining $\rho_{T}(x)$. Thus, rather than removing the issue of parametrization, it merely displaces it to $\rho_{T}(x)$. This density can itself be obtained by minimization of some appropriate functional (see (4.10) in section 4.1 containing numerical results).

The calculations above show some of the issues that arise when a maximum likelihood inference method is applied to estimate the drift (here $-\nabla V_{0}\left(X_{t}\right)$ ) in a diffusion (here (1.1)). They also uncover a connection between the maximum likelihood inference method often adopted by statisticians and the procedure of fitting $V$ to some 
empirical equilibrium density which is used by chemists and physicists. In the remainder of this paper we will generalize this connection. Specifically we note the following:

1. In section 2 , we will clean up the calculations above and prove the facts that we just listed. We will also outline how these calculations could be generalized to a generic time-reversible diffusion and indicate that a connection between the maximum likelihood inference and the procedure of fitting the drift to some empirical equilibrium density may exist in this case as well.

2. In section 3, we will generalize these conclusions to a specific nonreversible diffusion of great practical importance, namely, the Langevin equation, a hypo-elliptic diffusion process found by coupling a Hamiltonian system to a heat bath via white noise and damping.

3. In section 4, we will perform a series of numerical experiments to illustrate our results and discuss the following series of remaining issues: What is the influence of neglecting the boundary terms in (1.14)? What happens when the data is sampled at discrete times (in this case (1.6) and (1.9), and hence (1.6) and (1.11), are no longer equivalent)? What are the options to estimate $\rho_{T}(x)$ in (1.14)?

Section 5 contains some concluding remarks.

\section{Drift inference for time-reversible processes.}

2.1. Smoluchowski equation. In this section we make precise the results in the introduction.

First we analyze some properties of the log likelihood function $\mathcal{I}_{T}(V)$, written either as in (1.6) or (1.11). We start by stating a theorem which indicates that attempting to minimize (1.11) directly may be ill advised. We do this in the special case $d=1$ and where the domain of integration is restricted to $[0,1]$ and boundary terms are neglected, i.e., we consider the functional

$$
\mathcal{I}_{B}(b)=\int_{0}^{1}\left(b^{2}(x)-b^{\prime}(x)\right) \mu(d x)
$$

for $b \in H^{1}(0,1)$.

THEOREM 2.1. If $\mu(d x)$ in (2.1) is absolutely continuous with respect to Lebesgue measure with density given by a realization of the Brownian bridge, then the functional $\mathcal{I}_{B}(b)$ is a.s. not bounded below for $b \in H^{1}(0,1)$.

Proof. See the appendix.

While singular in the sense above when $T<\infty$, the log likelihood function $\mathcal{I}_{T}(V)$ has a nice limit as $T \rightarrow \infty$. To show this, we first make the following assumption which summarizes all the assumptions made so far on the SDE (1.1).

Assumption 1.

1. There exist $C_{1}, C_{2}>0$ such that for all $x \in \mathbb{R}^{d}$,

$$
C_{1}+\left\langle x, \nabla V_{0}(x)\right\rangle \geq C_{2}|x|^{2} .
$$

2. $V_{0}: \mathbb{R}^{d} \rightarrow \mathbb{R}$ is of class $C^{\infty}$, bounded from below and polynomially bounded from above for $|x| \rightarrow \infty$.

3. The inverse temperature is $\beta>0$.

TheOrem 2.2. Let Assumption 1 hold, and also assume that $V(x)$ is polynomially bounded and measurable. Then as $T \rightarrow \infty$, the functional $\mathcal{I}_{T}(V)$ in (1.6) converges a.s. to the functional $\mathcal{I}_{\infty}(V)$ defined in (1.8). 
This theorem is a consequence of the following lemma.

LEMMA 2.3. Under the assumptions of Theorem 2.2, (1.1) is ergodic with respect to the equilibrium measure with the density (1.2) and

$$
\lim _{T \rightarrow \infty} \frac{\sqrt{2 \beta}}{2 T} \int_{0}^{T}\left\langle\nabla V\left(X_{t}\right), d W_{t}\right\rangle=0 \quad \text { a.s. }
$$

Proof. The ergodicity follows from [16]. Thus, all the assumptions needed to apply Lemma 6.1 given in the appendix are satisfied.

Next we analyze the parametric log likelihood function (1.13) used in the parametric approach. We have the following.

TheOrem 2.4. Let Assumption 1 hold, and let $F=\left\{f_{i j}\right\}$ be the matrix with entries

$$
f_{i j}=\frac{1}{T} \int_{0}^{T}\left\langle\nabla f_{i}\left(X_{t}\right), \nabla f_{j}\left(X_{t}\right)\right\rangle d t, \quad i, j=1, \ldots, N,
$$

and assume that $F$ is positive definite. Then (1.13) has a unique minimizer. In addition, this minimizer is then given by

$$
\hat{\theta}=F^{-1} h,
$$

where $h$ is the vector with components

$$
h_{i}=-\frac{1}{T} \int_{0}^{T}\left\langle\nabla f_{i}\left(X_{t}\right), d X_{t}\right\rangle, \quad i=1, \ldots, N
$$

Furthermore, if the $\nabla f_{i}$ are polynomially bounded, then $\lim _{T \rightarrow \infty} F$ exists and is a.s. invertible.

Proof. The proof is immediate.

Finally, we analyze the properties of the approximate log likelihood function $\tilde{\mathcal{I}}_{T}(V)$ in (1.14) used in the nonparametric approach. Note that Theorem 5.5 of Chapter 2 in [14] implies that

$$
\limsup _{t \rightarrow \infty} \frac{\left|X_{t}\right|}{\sqrt{\log t}} \leq \sqrt{\frac{2 e}{C_{2} \beta}} \text { a.s. }
$$

An immediate consequence of this is that the boundary term in (1.14) is negligible.

TheOREm 2.5. Under Assumption 1 we have, for any $\varepsilon>0$, that

$$
\limsup _{t \rightarrow \infty} \frac{V\left(X_{t}\right)}{t^{\varepsilon}}=0 \quad \text { a.s. }
$$

The next theorem shows that the minimization problem associated with (1.15) has a unique solution as long as the density $\rho_{T}$ in this functional satisfies some requirements. To be able to state it more neatly, we introduce the space $\mathcal{V}$ as follows. For any open and bounded subset $U \subset \mathbb{R}^{d}$, define

$$
\mathcal{V}(U)=\left\{V \in H^{1}(U): \int_{U} V(x) d x=0\right\} .
$$

Theorem 2.6. Let $\rho_{T}: \mathbb{R}^{d} \rightarrow \mathbb{R}$ be smooth, $\rho_{T} \in C^{\infty}\left(\mathbb{R}^{d}\right)$. Furthermore, let $U$ be a bounded open subset of $\mathbb{R}^{d}$, and let $\rho_{T}$ be bounded below on $U: \exists \varepsilon>0$ for all $x \in U: \rho_{T}(x)>\varepsilon$. Then the minimizer of

$$
\inf _{V \in \mathcal{V}(U)} \frac{\beta}{4} \int_{U}\left(|\nabla V(x)|^{2}-2 \beta^{-1} \Delta V(x)\right) \rho_{T}(x) d x
$$

Copyright $@$ by SIAM. Unauthorized reproduction of this article is prohibited. 
is unique and given by

$$
\hat{V}(x)=-\beta^{-1} \log \rho_{T}(x)+C, \quad \text { where } \quad C=\beta^{-1} \int_{U} \rho_{T}(x) d x .
$$

The theorem can be proved using results from [5], but the proof can also be carried out by directly completing the square. The basic idea was given in the developments made in (1.16).

2.2. The generic time-reversible diffusion process. In this section, we assume that the data $\left\{X_{t}\right\}_{t \in[0, T]}$ has been generated by the following Itô SDE:

$$
d X_{t}=b_{0}\left(X_{t}\right) d t+\sigma_{0}\left(X_{t}\right) d W_{t}
$$

where $b_{0}: \mathbb{R}^{d} \rightarrow \mathbb{R}^{d}$ is the drift coefficient, $\sigma_{0}: \mathbb{R}^{d} \times \mathbb{R}^{d} \rightarrow \mathbb{R}^{d}$ is the diffusion coefficient, and $W_{t}$ is a standard $d$-dimensional Brownian motion. The diffusion coefficient $\sigma_{0}(x)$ is assumed to be known and we wish to estimate the drift $b_{0}(x)$. Additionally, we make the following assumption.

Assumption 2.

- Both $\sigma_{0}$ and $b_{0}(x)$ are $C^{1}\left(\mathbb{R}^{d}\right)$ and globally Lipschitz.

- We have

$$
\exists C>0 \forall x, \eta \in \mathbb{R}^{d}:\left\langle\eta, \sigma_{0} \sigma_{0}^{T}(x) \eta\right\rangle \geq C|\eta|^{2} .
$$

- The process $X_{t}$ is ergodic with invariant measure with density $\rho_{0}(x)$ with respect to Lebesgue measure; i.e., for every polynomially bounded measurable $f: \mathbb{R}^{d} \rightarrow \mathbb{R}$, we have that

$$
\lim _{T \rightarrow \infty} \frac{1}{T} \int_{0}^{T} f\left(X_{t}\right) d t=\int_{\mathbb{R}^{d}} f(a) \rho_{0}(a) d a \quad \text { a.s. }
$$

- Expected values of functions of the sample path converge to the invariant average exponentially; i.e., for every measurable polynomially bounded function $f$ there is a function $\Phi(\cdot)>0$ and $\lambda>0$ so that for almost any starting value $X_{0}$, we have

$$
\left|\mathbb{E}\left[f\left(X_{t}\right)\right]-\int_{\mathbb{R}^{d}} \rho_{0}(a) f(a) d a\right| \leq \Phi(x(0)) e^{-\lambda t} .
$$

- The process $X_{t}$ is time reversible.

We also proceed on the basis of the following conjecture, which is true under Assumption 1 as shown in Theorem 2.5, but in the more general context of Assumption 2 this is more difficult to establish.

Conjecture 1. For the process $X_{t}$ and under Assumption 2 the Stratonovich integral is a correction term which vanishes as $T \rightarrow \infty$ :

$$
\lim _{T \rightarrow \infty} \frac{1}{T} \int_{0}^{T}\left\langle b\left(X_{t}\right), \circ d X_{t}\right\rangle_{a_{0}\left(X_{t}\right)}=0 \quad \text { a.s. }
$$

A formal argument exploiting reversibility leads us to believe that this is true. This follows from the fact that the time reversibility assumption means that

$$
\begin{aligned}
& \left\{X_{t-T / 2}\right\}_{t \in[-T / 2, T / 2]} \text { and }\left\{X_{T / 2-t}\right\}_{t \in[-T / 2, T / 2]} \text { are equivalent in law } \\
& \text { in the limit as } T \rightarrow \infty \text {. }
\end{aligned}
$$

Copyright (C) by SIAM. Unauthorized reproduction of this article is prohibited. 
However, proof of Conjecture 1 appears nontrivial and is beyond the scope of this paper. The time reversibility also implies that $b_{0}(x), a_{0}(x)=\sigma_{0} \sigma_{0}^{T}(x)$, and $\rho_{0}(x)$ are related as

$$
0=b_{0} \rho_{0}-\frac{1}{2} \operatorname{div}\left(a_{0} \rho_{0}\right),
$$

which expresses the fact that a time-reversible process has no probability current at equilibrium. Note that since $\rho_{0}$ is unknown to us (only $\sigma_{0}$ and hence $a_{0}=\sigma_{0} \sigma_{0}^{T}$ are assumed to be available), (2.14) cannot be used a priori to determine $b_{0}$. Nevertheless, the nonparametric approach would be to simply approximate $\rho_{0}$ in (2.14) by some empirical density $\rho_{T}$ and thereby obtain an estimate for $b$. Next we show that this approach is closely related to the parametric approach in that both approaches correspond to minimizing a different regularization of the likelihood functional for $b_{0}$.

Proceeding as in the introduction, we can derive the negative of the log likelihood functional for the unknown drift $b$ given the data $\left\{X_{t}\right\}_{t \in[0, T]}$. Up to an irrelevant constant, this functional is

$$
\mathcal{I}_{T}(b)=\frac{1}{T} \int_{0}^{T}\left(\left|b\left(X_{t}\right)\right|_{a_{0}\left(X_{t}\right)}^{2} d t-2\left\langle b\left(X_{t}\right), d X_{t}\right\rangle_{a_{0}\left(X_{t}\right)}\right),
$$

where we introduced the following inner product and norm on the tangent space at $x \in \mathbb{R}^{d}$ :

$$
\begin{aligned}
\langle\eta, \xi\rangle_{a_{0}(x)} & =\left\langle\eta, a_{0}^{-1}(x) \xi\right\rangle & \forall \eta, \xi & \in \mathbb{R}^{d}, \\
|\eta|_{a_{0}(x)}^{2} & =\langle\eta, \eta\rangle_{a_{0}(x)} & \forall \eta & \in \mathbb{R}^{d} .
\end{aligned}
$$

This inner product and the norm are well defined since $a_{0}(x)$ is invertible at every $x \in \mathbb{R}^{d}$ by assumption (2.11).

As in (1.6), the log likelihood function (2.15) for $b$ is unbounded below in general if the data is finite, $T<\infty$. We can, however, proceed as for the Smoluchowski equation (1.1) along the following lines:

1. If we let $T \rightarrow \infty,(2.15)$ tends to a functional whose unique minimizer is $b_{0}$.

2. If we parametrize $b$ by the following form suggested by (2.14),

$$
b(x)=\frac{1}{2} \operatorname{div} a_{0}(x)-\frac{1}{2} a_{0}(x) \nabla V(x, \theta),
$$

with $V(x, \theta)$ as in (1.12) (thus $V(x, \theta)$ is approximating $\left.-\log \rho_{0}\right),(2.15)$ becomes a quadratic and convex function for $\theta=\left(\theta_{1}, \ldots, \theta_{N}\right)$ whose unique minimizer can be determined by solving a linear algebraic problem. This is the parametric approach.

3 . There is an alternative way to regularize $(2.15)$ which involves transforming the time integral in (2.15) into an expectation with respect to the occupation measure (1.10) and approximating $\mu_{T}(d x)$ by $\rho_{T}(x) d x$, where $\rho_{T}(x)$ is some smooth density. Then the minimizer of this regularized log likelihood function is unique and related to $\rho_{T}$ in the same way as $b_{0}$ is related to $\rho_{0}$ in (2.14). This is the nonparametric approach.

Let us analyze in more detail the statements made in these three points. The statement made in point 1 is a simple consequence of using (2.10) to rewrite (2.15) as

$$
\begin{aligned}
\mathcal{I}_{T}(b) & =\frac{1}{T} \int_{0}^{T}\left(\left|b\left(X_{t}\right)\right|_{a_{0}\left(X_{t}\right)}^{2}-2\left\langle b\left(X_{t}\right), b_{0}\left(X_{t}\right)\right\rangle_{a_{0}\left(X_{t}\right)}\right) d t \\
& -\frac{2}{T} \int_{0}^{T}\left\langle b\left(X_{t}\right), \sigma_{0}\left(X_{t}\right) d W_{t}\right\rangle_{a_{0}\left(X_{t}\right)} .
\end{aligned}
$$

Copyright $@$ by SIAM. Unauthorized reproduction of this article is prohibited. 
Under Assumption 2, Lemma 6.1 given in the appendix guarantees that the stochastic integral converges a.s. to zero; this is exactly what happens for the Smoluchowski equation (see Lemma 2.3). By ergodicity, the first integral converges a.s. toward an expectation with respect to the equilibrium distribution with density $\rho_{0}$. Thus, $\mathcal{I}_{T}(b)$ is expected to converge a.s. toward the functional $\mathcal{I}_{\infty}(b)$ given by

$$
\mathcal{I}_{\infty}(b)=\int_{\mathbb{R}^{d}}\left(|b(x)|_{a_{0}(x)}^{2}-2\left\langle b(x), b_{0}(x)\right\rangle_{a_{0}(x)}\right) \rho_{0}(x) d x .
$$

If $\rho_{0}(x)>0$, completing the square shows that the minimizer of this functional is unique and given by $b(x)=b_{0}(x)$, as needed. Of course, (2.19) is unavailable in practice since the data is finite.

Consider now the statement made in point 2. If we insert (2.17) into (2.15) and neglect all the irrelevant terms independent of $\theta$, as well as an overall multiplicative constant, we arrive at

$$
\mathcal{I}_{T}(\theta)=\theta^{T} \bar{F} \theta-2 \theta^{T} \bar{h},
$$

where $\bar{F}=\left\{\bar{f}_{i j}\right\}$ is the matrix with entries

$$
\bar{f}_{i j}=\frac{1}{T} \int_{0}^{T}\left\langle\nabla f_{i}\left(X_{t}\right), a_{0}\left(X_{t}\right) \nabla f_{j}\left(X_{t}\right)\right\rangle d t, \quad i, j=1, \ldots, N,
$$

and $\bar{h}$ is the vector with components

$$
\bar{h}_{i}=\frac{1}{T} \int_{0}^{T}\left(\left\langle\nabla f_{i}\left(X_{t}\right), \operatorname{div} a_{0}\left(X_{t}\right)\right\rangle d t-2\left\langle\nabla f_{i}\left(X_{t}\right), d X_{t}\right\rangle\right), \quad i=1, \ldots, N .
$$

This is a quadratic function in $\theta$ which is strictly convex iff the matrix $F$ is positive definite. If this is the case, (2.20) has a unique minimizer given by

$$
\theta=\bar{F}^{-1} \bar{h} .
$$

These results are equivalent to Theorem 2.4 except that they concern the process specified by (2.10) rather than the one specified by the Smoluchowski equation (1.1). Note that these results remain true even if the process defined by (2.10) is not time reversible, since (2.20) remains the parametric approximation via (2.17) of the negative $\log$ likelihood function for $b$ irrespective of whether the process is time reversible or not.

To establish the statements made in point 3 above, we will use the following relation between the Itô integral in (2.15) and the corresponding Stratonovich integral:

$$
\begin{aligned}
\int_{0}^{T}\left\langle b\left(X_{t}\right), d X_{t}\right\rangle_{a_{0}\left(X_{t}\right)} & =\int_{0}^{T}\left\langle b\left(X_{t}\right), \circ d X_{t}\right\rangle_{a_{0}\left(X_{t}\right)} \\
& +\frac{1}{2} \int_{0}^{T}\left(\left\langle b\left(X_{t}\right), \operatorname{div} a_{0}\left(X_{t}\right)\right\rangle_{a_{0}\left(X_{t}\right)}-\operatorname{div} b\left(X_{t}\right)\right) d t .
\end{aligned}
$$

Using this relation, as well as the occupation measure $\mu_{T}$ of the process $\left\{X_{t}\right\}_{t \in[0, T]}$, (2.15) can be written as

$$
\begin{aligned}
\mathcal{I}_{T}(b) & =\int_{\mathbb{R}^{d}}\left(|b(x)|_{a_{0}(x)}^{2}+\operatorname{div} b(x)-\left\langle b(x), \operatorname{div} a_{0}(x)\right\rangle_{a_{0}(x)}\right) \mu_{T}(d x) \\
& -\frac{2}{T} \int_{0}^{T}\left\langle b\left(X_{t}\right), \circ d X_{t}\right\rangle_{a_{0}\left(X_{t}\right)} .
\end{aligned}
$$

Copyright $\odot$ by SIAM. Unauthorized reproduction of this article is prohibited. 
The stochastic integral in this expression is a correction term which we expect will vanish in the limit as $T \rightarrow \infty$, as stated in Conjecture 1 . Thus, if we assume that $T$ is large enough so that we can neglect the stochastic integral term in (2.25), and we approximate the occupation measure $\mu_{T}(x)$ by $\rho_{T}(x) d x$, where $\rho_{T}(x)$ is a smooth density with bounded support, we can approximate the log likelihood function (2.25) by

$$
\tilde{\mathcal{I}}_{T}(b)=\int_{\mathbb{R}^{d}}\left(|b(x)|_{a_{0}(x)}^{2}+\operatorname{div} b(x)-\left\langle b(x), \operatorname{div} a_{0}(x)\right\rangle_{a_{0}(x)}\right) \rho_{T}(x) d x .
$$

This functional is the equivalent of the expression (1.15) when considering (2.10) instead of the Smoluchowski equation (1.1). Given the smoothness of the density, we can perform the following partial integration:

$$
\tilde{\mathcal{I}}_{T}(b)=\int_{\mathbb{R}^{d}}\left(|b(x)|_{a_{0}(x)}^{2} \rho_{T}(x)-b(x) \cdot \nabla \rho_{T}(x)-\left\langle b(x), \operatorname{div} a_{0}(x)\right\rangle_{a_{0}(x)} \rho_{T}(x)\right) d x,
$$

where the boundary terms vanish since $\rho_{T}(\cdot)$ has bounded support. This functional has much nicer properties than the original $\mathcal{I}_{T}(b)$ in $(2.15)$, as shown by the following result.

TheOrem 2.7. Let Assumption 2 hold. Also, let $U$ be a bounded open subset of $\mathbb{R}^{d}$, and assume that $\rho_{T} \in \mathcal{C}^{\infty}(U)$ is bounded below on $U: \exists \varepsilon>0: \rho_{T}(x)>\varepsilon$ for all $x \in U$. Then for the functional

$$
\tilde{\tilde{\mathcal{I}}}_{T}(b)=\int_{U}\left(|b(x)|_{a_{0}(x)}^{2} \rho_{T}(x)-b(x) \cdot \nabla \rho_{T}(x)-\left\langle b(x), \operatorname{div} a_{0}(x)\right\rangle_{a_{0}(x)} \rho_{T}(x)\right) d x
$$

the minimizer of

$$
\inf _{b \in L^{2}(U)} \tilde{\tilde{\mathcal{I}}}_{T}(b)
$$

is unique and given by

$$
\tilde{b}=\frac{1}{2} \operatorname{div}\left(a_{0} \rho_{T}\right) / \rho_{T} \quad(x \in U) .
$$

Proof. Rewrite the functional $\tilde{\tilde{\mathcal{I}}}$, introducing an extra factor of $\rho_{T}$ and $a_{0}$ in the middle term to recognize it as a quadratic form in $b$ :

$\tilde{\tilde{\mathcal{I}}}(b)=\int_{U}\left(|b(x)|_{a_{0}(x)}^{2}-\left\langle b(x), a_{0}(x) \frac{\nabla \rho_{T}(x)}{\rho_{T}(x)}\right\rangle_{a_{0}(x)}-\left\langle b(x), \operatorname{div} a_{0}(x)\right\rangle_{a_{0}(x)}\right) \rho_{T}(x) d x$.

Now complete the square to obtain

$$
\begin{aligned}
\tilde{\tilde{\mathcal{I}}}(b)=\int_{U} & \left(\left|b(x)-\frac{a_{0}(x)}{2} \frac{\nabla \rho_{T}(x)}{\rho_{T}(x)}-\frac{1}{2} \operatorname{div} a_{0}(x)\right|_{a_{0}(x)}^{2}\right. \\
& \left.-\left|\frac{a_{0}(x)}{2} \frac{\nabla \rho_{T}(x)}{\rho_{T}(x)}-\frac{1}{2} \operatorname{div} a_{0}(x)\right|_{a_{0}(x)}^{2}\right) \rho_{T}(x) d x .
\end{aligned}
$$

Since $\rho_{T}(\cdot)$ is strictly positive on $U$, this functional is minimized when

$$
0=b-\frac{a_{0}(x)}{2} \frac{\nabla \rho_{T}}{\rho_{T}}-\frac{1}{2} \operatorname{div} a_{0} \quad(x \in U) .
$$

Copyright (c) by SIAM. Unauthorized reproduction of this article is prohibited. 
This is an algebraic equation for $b$ whose solution is (2.29).

Relation (2.29) is the equivalent of (1.17) for a generic time-reversible process and shows how the nonparametric approach of deducing the drift coefficient from the equilibrium density and the diffusion coefficient can be generalized to this case.

An interesting consequence of the calculations above is that the time-ordering of the data is not very relevant for time-reversible processes. This is clear for the nonparametric approach based on (2.26) and leading to (2.29) in which only the empirical density $\rho_{T}(x)$ plays a role. Similarly, we expect that time-ordering plays only a small role in the parametric approach based on regularizing the maximum likelihood function leading to (2.20) via parametrization of the drift $b$. This conjecture will be verified in the numerical experiments of section 4 .

3. Nonreversible processes: The Langevin equation. The calculations in section 2 rely heavily on the property that the process is time reversible. In particular, for a nonreversible process, we would not expect Conjecture 1 to hold in general; hence we will not be able to approximate the log likelihood function by (2.26) (in which the contribution from the stochastic integral term in (2.25) is missing). Another way to look at the problem is to realize that, for a nonreversible process, relation (2.14) is replaced by

$$
j_{0}(x)=b_{0} \rho_{0}-\frac{1}{2} \operatorname{div}\left(a_{0} \rho_{0}\right),
$$

where $j_{0}(x)$ is a divergence-free vector field accounting for the nonzero equilibrium probability current of the nonreversible process. Equation (3.1) implies that it is not straightforward to generalize the nonparametric approach to nonreversible processes since, on top of the diffusion tensor $a_{0}$ and the equilibrium density $\rho_{0}$ (or some approximations thereof), we need an approximation of the current $j_{0}$ to deduce the drift $b_{0}$. This approximation of $j_{0}$ will not be available in general. Despite all this, in this section we show that the nonparametric approach can be generalized to a specific type of nonreversible process which frequently arises in applications, and that this approach is again closely connected to the parametric approach for these processes. The specific type of nonreversible process is that governed by the Langevin equation:

$$
\ddot{Q}_{t}+\beta_{0} D_{0} \dot{Q}_{t}+\nabla V_{0}\left(Q_{t}\right)=\sqrt{2 D_{0}} \dot{W}_{t},
$$

where $\beta_{0}$ is the inverse temperature, $D_{0}$ is the diffusivity, and $W_{t}$ is a standard Brownian motion. (Thus the friction coefficient $\gamma$ is related to $\beta_{0}$ and $D_{0}$ via the Einstein relation: $D_{0}=\gamma / \beta_{0}$.) We assume that $D_{0}$ is known, and we wish to find the potential $V_{0}$ and the inverse temperature $\beta_{0}$.

If we set $P_{t}=\dot{Q}_{t}\left(Q_{t}\right.$ is referred to as position, $P_{t}$ as momentum), then from (3.2) we obtain the following system of equations:

$$
\left\{\begin{array}{l}
\dot{Q}_{t}=P_{t}, \\
\dot{P}_{t}=-\beta_{0} D_{0} P_{t}-\nabla V_{0}\left(Q_{t}\right)+\sqrt{2 D_{0}} \dot{W}_{t} .
\end{array}\right.
$$

Note that since the noise enters the equation only for $P_{t},(3.3)$ does not define an elliptic diffusion; it is, however, hypo-elliptic; see [16]. If one assumes that $V_{0}$ satisfies the assumptions in Theorem 2.2, the process generated by (3.3) is ergodic with respect to the equilibrium distribution with density

$$
\rho_{0}(q, p)=\rho_{0}(q) g_{0}(p),
$$


where

$$
\rho_{0}(q)=Z^{-1} e^{-\beta_{0} V_{0}(q)}, \quad g_{0}(q)=\left(2 \pi \beta_{0}\right)^{-d / 2} e^{-\frac{1}{2} \beta_{0}|p|^{2}} .
$$

Note in particular that the equilibrium distribution is Gaussian in the momentum coordinate.

The Radon-Nikodym derivative of the measure on path-space for (3.3) with respect to the measure generated by

$$
\dot{P}_{t}=\sqrt{2 D_{0}} \dot{W}_{t}
$$

is given by

$$
\exp \left(-\frac{T}{2 D_{0}} I_{T}(Q, P)\right)
$$

Here

$$
I_{T}(Q, P)=\frac{1}{2 T} \int_{0}^{T}\left(\left|\beta_{0} D_{0} P_{t}+\nabla V_{0}\left(Q_{t}\right)\right|^{2} d t+2\left\langle\beta_{0} D_{0} P_{t}+\nabla V_{0}\left(Q_{t}\right), d P_{t}\right\rangle\right),
$$

where it is understood that $Q_{t}$ and $P_{t}$ are related as $\dot{Q}_{t}=P_{t}$, as in (3.3). For fixed data $\left\{Q_{t}, P_{t}\right\}_{t \in[0, T]}$, we may evaluate (3.8) at $V$ and $\beta$ differently from $V_{0}$ and $\beta_{0}$. The resulting functional is then the negative of the $\log$ likelihood function for $V$ and $\beta$ :

$$
\mathcal{I}_{T}(V, \beta)=\frac{1}{2 T} \int_{0}^{T}\left(\left|\beta D_{0} P_{t}+\nabla V\left(Q_{t}\right)\right|^{2} d t+2\left\langle\beta D_{0} P_{t}+\nabla V\left(Q_{t}\right), d P_{t}\right\rangle\right) .
$$

As in the Smoluchowski case, the log likelihood function (3.9) must be regularized to be useful. The simplest way is to parametrize $V(q)$ as in (1.12), in which case (3.9) reduces to a function of $\beta$ and $\theta=\left(\theta_{1}, \ldots, \theta_{N}\right)$ which can then be minimized over these parameters. This is the parametric approach. Next we investigate another type of regularization of (3.9) leading to the equivalent of the nonparametric approach.

We begin by making a few transformations on (3.9). First, notice that an integration by parts using the Itô formula and $\dot{Q}_{t}=P_{t}$ shows that

$$
\begin{aligned}
\int_{0}^{T}\left\langle\nabla V\left(Q_{t}\right), d P_{t}\right\rangle & =-\int_{0}^{T}\left\langle P_{t}, \nabla \nabla V\left(Q_{t}\right) P_{t}\right\rangle d t+\left[\left\langle\nabla V\left(Q_{t}\right), P_{t}\right\rangle\right]_{0}^{T} \\
\text { and } \int_{0}^{T}\left\langle P_{t}, d P_{t}\right\rangle & =\frac{1}{2}\left[\left|P_{t}\right|^{2}\right]_{0}^{T}-d D_{0} T .
\end{aligned}
$$

Thus

$$
\begin{aligned}
\mathcal{I}_{T}(V, \beta)= & \frac{1}{T}\left[\beta D_{0}\left|P_{t}\right|^{2}+\left\langle\nabla V\left(Q_{t}\right), P_{t}\right\rangle\right]_{0}^{T} \\
& -d D_{0}^{2} \beta+\frac{1}{2 T} \int_{0}^{T}\left(\left|\beta D_{0} P_{t}+\nabla V\left(Q_{t}\right)\right|^{2}-2\left\langle P_{t}, \nabla \nabla V(q) P_{t}\right\rangle\right) d t .
\end{aligned}
$$

Under suitable conditions on the potentials $V_{0}$ and $V$, the boundary contributions from the two integrations by parts converge a.s. to zero as $T \rightarrow \infty$, as made precise in the following lemma.

Lemma 3.1. Assume that $V \in C^{1}\left(\mathbb{R}^{d}, \mathbb{R}+\right)$ and that $\exists C_{i}>0, i=1, \ldots, 5$, where $C_{1}<1$ and $m \in \mathbb{Z}^{+}$such that

Copyright $($ C by SIAM. Unauthorized reproduction of this article is prohibited. 
- $\frac{1}{2}\left\langle\nabla V_{0}(q), q\right\rangle \geq C_{1} V_{0}(q)+C_{2}|q|^{2}-C_{3}$ for all $q \in \mathbb{R}^{d}$,

- $|\nabla V(q)| \leq C_{4}\left[1+|q|^{2 m-1}\right]$ for all $q \in \mathbb{R}^{d}$,

- $\left|\nabla V_{0}(q)\right| \leq C_{5}\left[1+|q|^{2 m-1}\right]$ for all $q \in \mathbb{R}^{d}$.

Then there is a $C>0$ such that

$$
\limsup _{t \rightarrow \infty} \frac{\left|P_{t}\right|^{2}+\left|Q_{t}\right|^{2}}{\log t} \leq C \quad \text { a.s. }
$$

and

$$
\limsup _{t \rightarrow \infty} \frac{\left|\left\langle\nabla V\left(Q_{t}\right), P_{t}\right\rangle\right|+\left|P_{t}\right|^{2}}{t}=0 \quad \text { a.s. }
$$

Proof. Let $H(q, p)$ denote the following perturbed Hamiltonian:

$$
H(q, p)=\frac{1}{2}|p|^{2}+V(q)+D_{0} \beta_{0}\langle p, q\rangle+D_{0}^{2} \beta_{0}^{2}|q|^{2}+1 .
$$

Then

$$
H(q, p) \geq 1+\frac{1}{8}|p|^{2}+\frac{D_{0}^{2} \beta_{0}^{2}}{3}|q|^{2} .
$$

The arguments in section 3 of [16] show that there exist $\xi_{6}, \xi_{7}, \xi_{8}, \xi_{9}>0$ such that, for the generator $\mathcal{L}$ of $(3.3)$,

$$
\mathcal{L} H \leq \xi_{6}-\xi_{7} H
$$

and

$$
\left|\left\langle\nabla H,\left(\begin{array}{r}
0 \\
\sqrt{D_{0}}
\end{array}\right)\right\rangle\right|^{2} \leq \xi_{8}[|p|+|q|]^{2} \leq \xi_{9} H(q, p) .
$$

Thus, applying the Itô formula to $e^{\xi_{7} t} H(q(t), p(t))$ and using arguments similar to those in Theorem 5.5 of Chapter 2 in [14], but applied to $H(q, p)$ instead of $|p|^{2}+|q|^{2}$, give the first result. The second result follows since $\nabla V(q)$ is assumed polynomially bounded.

The ergodicity of the process, together with Lemma 3.1, implies that as $T \rightarrow \infty$, $\mathcal{I}_{T}(V, \beta)$ converges a.s. to the functional $\mathcal{I}_{\infty}(V, \beta)$ given by

$$
\mathcal{I}_{\infty}(V, \beta)=-d D_{0}^{2} \beta+\frac{1}{2} \int_{\mathbb{R}^{d} \times \mathbb{R}^{d}}\left(\left|\beta D_{0} p+\nabla V(q)\right|^{2}-2\langle p, \nabla \nabla V(q) p\rangle\right) \rho_{0}(q, p) d q d p .
$$

Using the fact that $\rho_{0}(q, p)$ is a product of two densities, $\rho_{0}(q, p)=\rho_{0}(q) g_{0}(p)$, and that $g_{0}(p)$ is Gaussian, the integral over the momentum in (3.11) can be performed explicitly. The result can be written as

$$
\mathcal{I}_{\infty}(V, \beta)=\frac{1}{2} \int_{\mathbb{R}^{d}}\left(|\nabla V(q)|^{2}-2 \beta_{0}{ }^{-1} \Delta V(q)\right) \rho_{0}(q) d q+d D_{0}{ }^{2}\left(\frac{1}{2} \beta^{2} / \beta_{0}-\beta\right)
$$

The integral on the right-hand side is, up to an irrelevant constant, the same as the one in (1.15) and it is the only term involving $V$. As a result, the minimum of (3.12) over $V$ is reached when $V=V_{0}+C$, where $C$ is an arbitrary constant, as in section 2.1.

Copyright $@$ by SIAM. Unauthorized reproduction of this article is prohibited. 
Similarly, the last term in (3.12) is minimized when $\beta=\beta_{0}$. Thus we conclude that, in the limit as $T \rightarrow \infty$, the log likelihood function for $V_{0}$ and $\beta_{0}$ has these parameters as unique maximizers.

When $T$ is finite, however, we need to proceed differently. First, we can replace the time integral in (3.10) by an expectation with respect to the occupation measure of the process $\left\{Q_{t}, P_{t}\right\}_{t \in[0, T]}$ :

$$
\begin{aligned}
& \mathcal{I}_{T}(V, \beta)=\frac{1}{T}\left[\beta D_{0}\left|P_{t}\right|^{2}+\left\langle\nabla V\left(Q_{t}\right), P_{t}\right\rangle\right]_{0}^{T} \\
& \quad-d D_{0}^{2} \beta+\frac{1}{2} \int_{\mathbb{R}^{d} \times \mathbb{R}^{d}}\left(\left|\beta D_{0} p+\nabla V(q)\right|^{2}-2\langle p, \nabla \nabla V(q) p\rangle\right) \mu_{T}(d q, d p) .
\end{aligned}
$$

Assuming that $T$ is large enough so that we can neglect the boundary terms in (3.10), we are left with the terms on the second line in (3.13). To regularize them, we must regularize $\mu_{T}(d q, d p)$ by some $\rho_{T}(q, p) d q d p$. Consistent with (3.4), we assume that the empirical density $\rho_{T}(q, p)$ factorizes as $\rho_{T}(q, p)=\rho_{T}(q) g_{T}(p)$, where $\rho_{T}(q)$ and $g_{T}(p)$ are densities which can be estimated separately by splitting the data into $\left\{Q_{t}\right\}_{t \in[0, T]}$ and $\left\{P_{t}\right\}_{t \in[0, T]}$. Consistent with (3.5), we can further assume that $g_{T}(p)$ is a Gaussian density of the form

$$
g_{T}(p)=\left(2 \pi \beta_{T}\right)^{-d / 2} e^{-\frac{1}{2} \beta_{T}|p|^{2}}
$$

where $\beta_{T}>0$ is a parameter which can be estimated from the data as

$$
\beta_{T}^{-1}=\frac{1}{d T} \int_{0}^{T}\left|P_{t}\right|^{2} d t
$$

Substituting $\rho_{T}(q, p) d q d p$ for $\mu_{T}(d q, d p)$ in the integral term in (3.13) and using (3.14), the integral over the momentum can be performed explicitly. This gives the following approximation for the terms on the second line in (3.13):

$$
\frac{1}{2} \int_{\mathbb{R}^{d}}\left(|\nabla V(q)|^{2}-2 \beta_{T}^{-1} \Delta V(q)\right) \rho_{T}(q) d q+d D_{0}{ }^{2}\left(\frac{1}{2} \beta^{2} / \beta_{T}-\beta\right) .
$$

This functional is similar to (3.11), except that it involves the empirical $\rho_{T}$ and $\beta_{T}$ instead of the actual $\rho_{0}$ and $\beta_{0}$. The following theorem is thus analogous to Theorem 2.6.

Theorem 3.2. Let $U \subset \mathbb{R}^{d}$ be open and bounded. Suppose that $\rho_{T}$ is bounded below on $U$, i.e., $\exists \varepsilon>0$ for all $x \in U: \rho_{T}(x)>\varepsilon$ holds. Assume furthermore that $\beta_{T}>0$. Then the functional

$$
\tilde{\tilde{I}}_{h}(V, \beta)=\frac{1}{2} \int_{U}\left(|\nabla V(q)|^{2}-2 \beta_{T}^{-1} \Delta V(q)\right) \rho_{T}(q) d q+d D_{0}^{2}\left(\frac{1}{2} \beta^{2} / \beta_{T}-\beta\right)
$$

has a unique minimizer $(V, \beta)$ in $\bar{H}^{1}(U) \times \mathbb{R}$, where the bar denotes functions of mean zero. This minimizer is given by

$$
\hat{V}=-\beta_{T}^{-1} \log \rho_{T}(x)+C, \quad \hat{\beta}=\beta_{T},
$$

where the constant $C$ is such as to ensure that $\hat{V}$ has mean zero.

Proof. First establish that $\hat{\beta}=\beta_{T}$, which is straightforward as $\beta$ occurs only in the second term. The rest of the proof proceeds analogously to Theorem 2.6.

Thus, the nonparametric approach can be generalized to the Langevin equation and leads to the fitting of $V$ to the empirical measure, similarly to what we found in the case of the Smoluchowski equation. Furthermore, the inverse temperature $\beta$ is estimated from the variance of the momentum in the empirical measure.

Copyright $@$ by SIAM. Unauthorized reproduction of this article is prohibited. 


\section{Numerical experiments.}

4.1. Setup. In this section we perform a series of numerical experiments on a simple model system to illustrate the results obtained in the previous sections, in particular the relationship between the practitioners' and statisticians' approaches to drift estimation. These experiments will also allow us to investigate two issues that we have left open so far. The first is the impact on the parametric approach of having a data set sampled at discrete points in time rather than continuously. The second issue is how to obtain the approximate density $\rho_{T}(x)$ needed in the nonparametric approach. The model system we will investigate is the one-dimensional diffusion

$$
\dot{X}_{t}=-X_{t}^{3}+\frac{3}{2} X_{t}+\frac{3}{2} \dot{W}_{t}, \quad X_{0}=0 .
$$

This equation is a special case of the Smoluchowski equation (1.1) with

$$
V_{0}(x)=\frac{1}{4} x^{4}-\frac{3}{4} x^{2}
$$

and $\beta=8 / 9$. To generate the data, we integrate (4.1) using the Euler-Maruyama scheme with time-step $\Delta t$ for $N_{T}=\lfloor T / \Delta t\rfloor$ steps, i.e., using

$$
X_{(j+1) \Delta t}=X_{j \Delta t}-X_{j \Delta t}^{3} \Delta t+\frac{3}{2} X_{j \Delta t} \Delta t+\frac{3}{2} \sqrt{\Delta t} \xi_{j}, \quad j=0, \ldots, N_{T}-1,
$$

with $X_{0}=0$ and where $\left\{\xi_{j}\right\}_{j=0, \ldots, N_{T}-1}$ are independent Gaussian variables with mean 0 and variance 1 . The value of $\Delta t$ and $T$ will be varied to measure the impact of these parameters. The Euler-Maruyama scheme produces a discrete time sample $\left\{X_{j \Delta t}\right\}_{j=0, \ldots, N_{T}}$ which we will use as data. For simplicity, we will denote this data set as $\left\{X_{j}\right\}_{j=0, \ldots, N_{T}}$.

In the parametric approach we use the following polynomial representation of the force $b_{0}(x)=-V_{0}^{\prime}(x)=-x^{3}+\frac{3}{2} x$ :

$$
b(x, \theta)=\sum_{i=0}^{3} \theta_{i} x^{i} .
$$

Equivalently, this means that we parametrize the potential $V_{0}(x)$ as

$$
V(x, \theta)=\sum_{i=0}^{3} \frac{\theta_{i} x^{i+1}}{i+1}
$$

Based on this parametrization, and consistent with the time-discretization used in (4.3), we adopt the following discretized version of the log likelihood function (1.13):

$$
\mathcal{I}_{T}(\theta)=\frac{1}{T} \sum_{j=0}^{N}\left(\left|b\left(X_{j}, \theta\right)\right|^{2} \Delta t-2 b\left(X_{j}, \theta\right)\left(X_{j+1}-X_{j}\right)\right) .
$$

The minimization of (4.6) gives rise to a linear algebraic system for $\theta=\left(\theta_{0}, \ldots, \theta_{3}\right)$ which is easy to solve (the solution is similar to (2.23) in the continuously sampled case). We refer to this solution as the MLE $\hat{\theta}$. 
In the nonparametric approach the main issue is the evaluation of the empirical density $\rho_{T}(x)$ in (1.15) and (1.17). To obtain results that can be easily compared with those of the parametric approach we will parametrize $\rho_{T}$ as

$$
\rho_{T}(x, \theta)=Z^{-1}(\theta) e^{-\beta V(x, \theta)}, \quad \text { where } \quad Z(\theta)=\int_{\mathbb{R}} e^{-\beta V(x, \theta)} d x,
$$

and $\beta=8 / 9$ is given. To then determine $\rho_{T}(x, \theta)$, we test and compare three different methods. The first method is based on estimating a discretization of the empirical density obtained by a standard histogram method using an even number $K$ of bins centered at $c_{k}=8 k / K$ for $k=-K / 2, \ldots, K / 2$. The bins are spaced equidistantly and the small number of samples outside $[-4,4]$ is discarded. Denoting by $\hat{\rho}_{k}$ this empirical density, we then obtain $\theta=\left(\theta_{0}, \ldots, \theta_{3}\right)$ by minimizing

$$
\sum_{k=-K / 2}^{K / 2}\left|\log \hat{\rho}_{k}+\beta V\left(c_{k}, \theta\right)\right|^{2} .
$$

This objective function is the discrete analogue of the $L^{2}$ norm of the difference between $-\beta V(x, \theta)$ and the log of a (putative) continuous approximation of the empirical density $\rho_{k}$. Note that this is a straightforward least squares problem of dimension $K$, so this is easily solved by standard methods. We refer to optimizing (4.8) as the practitioners' method, and call $\hat{\theta}$ optimizing (4.8) the practitioners' method estimator (PME).

For the second method, note that in one dimension the occupation measure $\mu_{T}$ has the scaled local time $L_{T}(x) / T$ as density, so one can search the minimizer of

$$
\int_{\mathbb{R}}\left|\rho_{T}(x, \theta)-L_{T}(x) / T\right|^{2} d x,
$$

which measures the $L^{2}$ distance between $\rho_{T}(x, \theta)$ and the scaled local time $L_{T}(x) / T$.

To adapt this to time-discrete observations, it is possible to expand the square and then approximate the local time as

$$
L_{T}=\frac{T}{N_{T}} \sum_{j=0}^{N_{T}} \delta_{X_{j}}
$$

This results in estimation via minimizing the following objective function over $\theta$ :

$$
\int_{\mathbb{R}} \rho_{T}^{2}(x, \theta) d x-\frac{2}{T} \sum_{j=0}^{N_{T}} \rho_{T}\left(X_{j}, \theta\right) .
$$

The third method is based on a coarsened version of (4.10) in which we use $\hat{\rho}_{k}$ to replace (4.10) by

$$
\sum_{k=-K}^{K} \rho_{T}^{2}\left(c_{k}, \theta\right)-2 \rho_{T}\left(c_{k}, \theta\right) \hat{\rho}_{k} .
$$

Minimizing (4.11) is slightly less accurate than minimizing (4.10), but it is computationally less expensive if the number of bins is significantly smaller than the number 


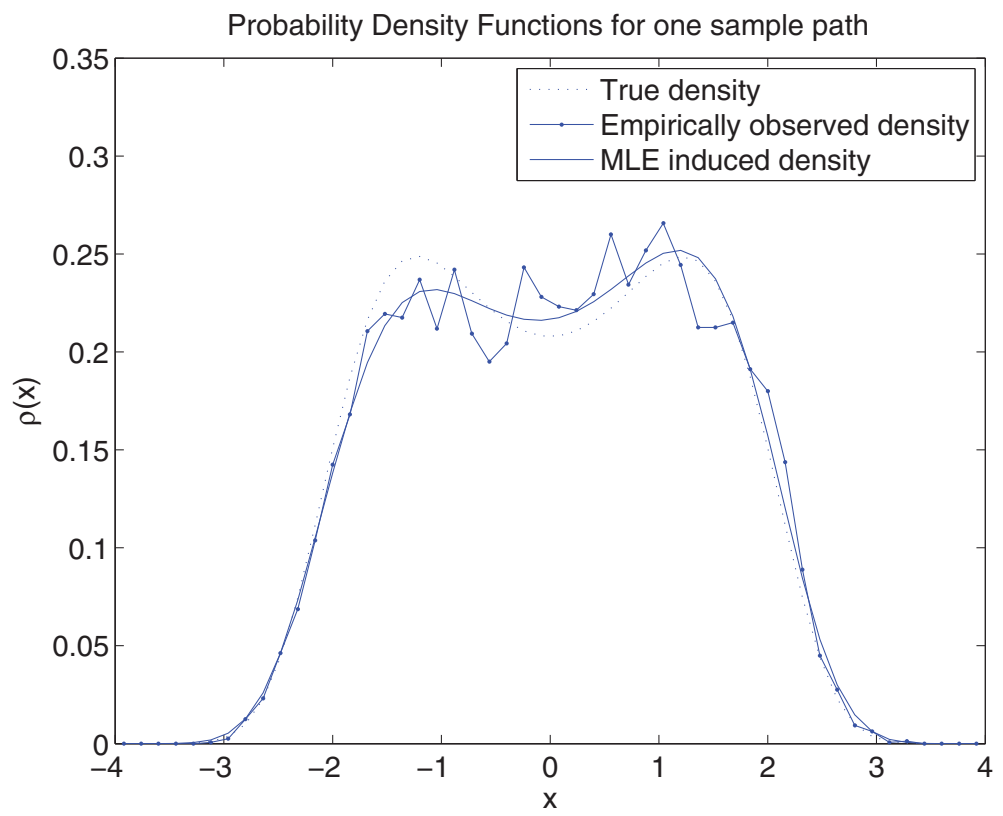

FIG. 4.1. Densities from one particular sample path.

of data points in the time-series, $K \ll N_{T}$. The computational cost involved in minimizing (4.10) compels us to use (4.11), but we study its behavior for several choices of $K$, the number of bins in the histogram. To optimize (4.11) we use steepest descent together with a line search strategy and refer to the optimal $\hat{\theta}$ as the minimum distance estimator (MDE).

More generally, using a histogram as a means of summarizing the data not only smoothes the empirical density but also makes optimization easier. In the case of the estimator (4.8), it is even unclear how this estimator could be used with the unsmoothed discrete time empirical density. Various alternative ways of obtaining a smoothed empirical density $\hat{\rho}$ from the discrete time observations $X_{j}$ are conceivable. Established methods include kernel density estimators and even nonparametric density estimation.

4.2. Connections via correlation. In order to establish that the link between the MLE (obtained from (4.6)) and the PME (obtained from (4.8)) persists for discretely observed data, we wish to study the stochastic dependency between the PME and the MLE understood as random variables.

Having verified that asymptotic unbiasedness and a suitable decay of variance are indeed observed for our implementation of these estimators, we consider that these results are standard at least for the MLE, so we do not show them here in detail.

Since applied interest resides in the invariant density and the empirical measure, it seems interesting to first compare the MLE and density-based estimators at the level of densities. To do this, we perform numerical simulations using $K=50$ bins for a final time of $T=100$ (and $\Delta t=0.01$ ) and compute the invariant density $\rho(\hat{\theta}, \cdot)$ induced by MLE estimates $\hat{\theta}$ of $\left\{\theta_{i}\right\}_{i=0}^{3}$. A typical case is shown in Figure 4.1, and repeated experiments computing the binwise correlation of deviations from the true 


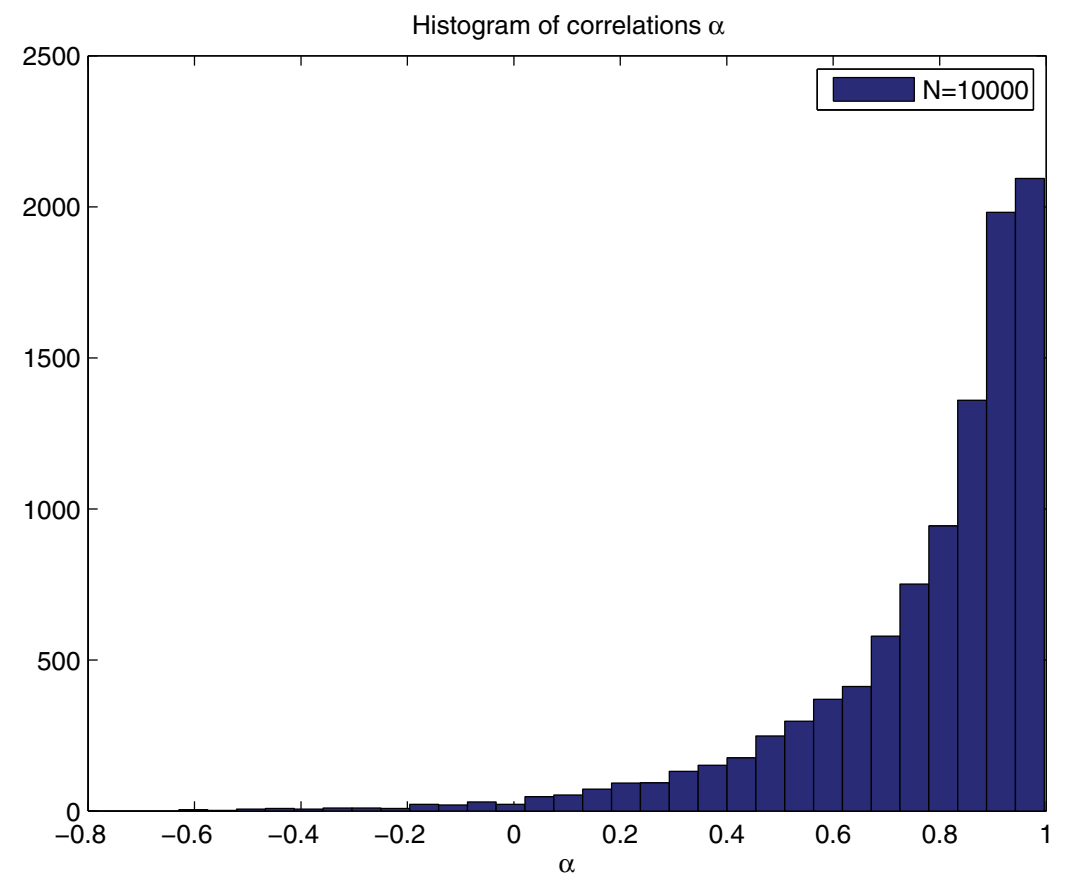

FIG. 4.2. Correlation coefficients $\alpha$ for deviations of MLE-induced and empirical densities from the invariant density.

invariant density $\rho$ (whose evaluation at $c_{k}$ we denote by $\rho_{k}=\rho\left(c_{k}\right)$ ), namely,

$$
\alpha=\frac{\sum_{k=-K / 2}^{K / 2}\left(\hat{\rho}_{k}-\rho_{k}\right) \cdot\left(\rho\left(\hat{\theta}, c_{k}\right)-\rho_{k}\right)}{\sqrt{\sum_{k=-K / 2}^{K / 2}\left(\hat{\rho}_{k}-\rho_{k}\right)^{2}} \cdot \sqrt{\sum_{k=-K / 2}^{K / 2}\left(\rho\left(\hat{\theta}, c_{k}\right)-\rho_{k}\right)^{2}}},
$$

show high correlations, as visible in the histogram in Figure 4.2. An MDE or PME that now attempts to fit the empirical density $\hat{\rho}$ or its logarithm using some least squares method would hence be expected to yield drift parameter estimates $\hat{\theta}$, whose deviations from $\theta$ are correlated with the MLE estimates' deviations.

To investigate whether this is so, it is useful to note the experimental observation that all three estimators display an approximately Gaussian distribution. We use the final time $T=160$ and the time-step $\Delta t=0.002$, and the MDE and PME each use $K=50$ bins throughout. We evaluate $N=1000$ realizations each of the MDE, MLE, and PME to produce estimates of $\left\{\theta_{3}^{(k)}\right\}_{k=1}^{N}$ of $\theta_{3}$. We then standardize these estimates, subtracting the mean and dividing by the standard error. Histograms and quantile-quantile plots of these three parameter estimates are given in Figures 4.3, 4.4, and 4.5, respectively. Furthermore, we apply a Kolmogorov-Smirnov test of normality and report the obtained $p$-values in these figures. In all three cases, the observed $p$ value is above $p=0.88$ so that the observed evidence against normality using the Kolmogorov-Smirnov test statistic is considered very weak. It should be pointed out that for smaller final times, the distribution of parameter estimates does not approximate a Gaussian as closely as this; theorems on (local) asymptotic normality that can be found for the MLE and MDE in continuous time, e.g., in [12], suggest only normality for large final times. 

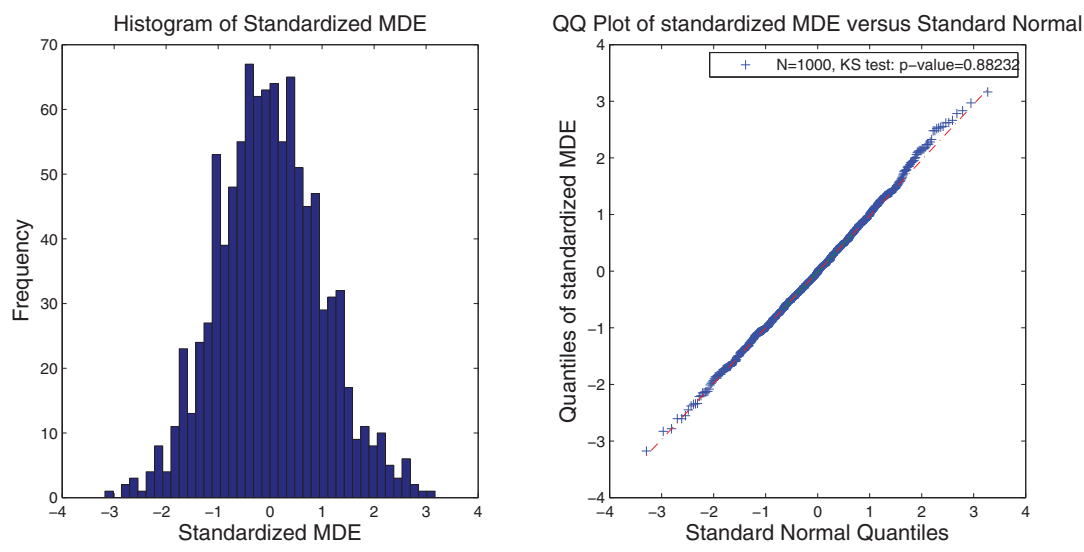

FIG. 4.3. Test of normality for the MDE.
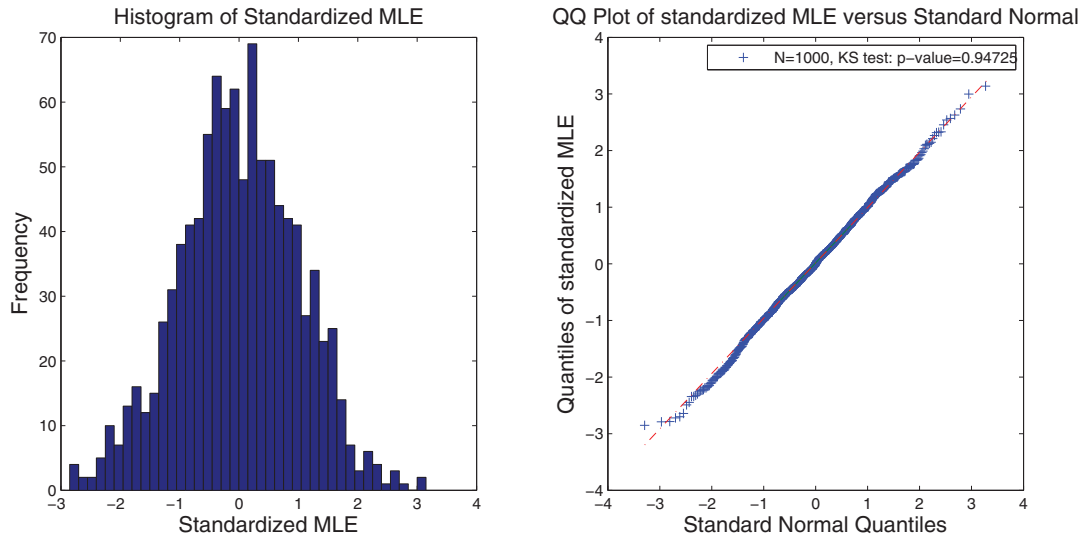

FIG. 4.4. Test of normality for the MLE.
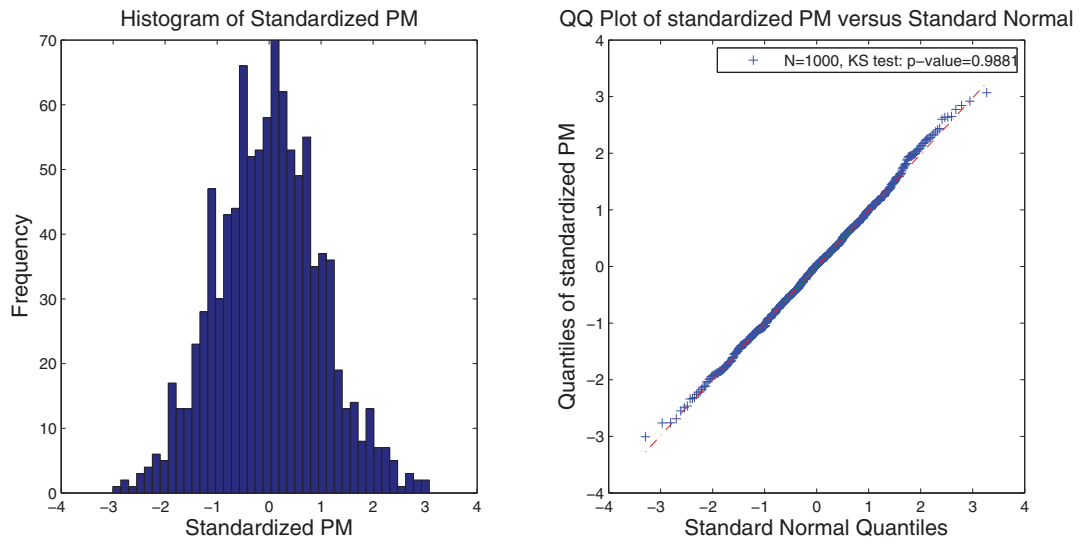

FIG. 4.5. Test of normality for the PME.

Copyright (C) by SIAM. Unauthorized reproduction of this article is prohibited. 


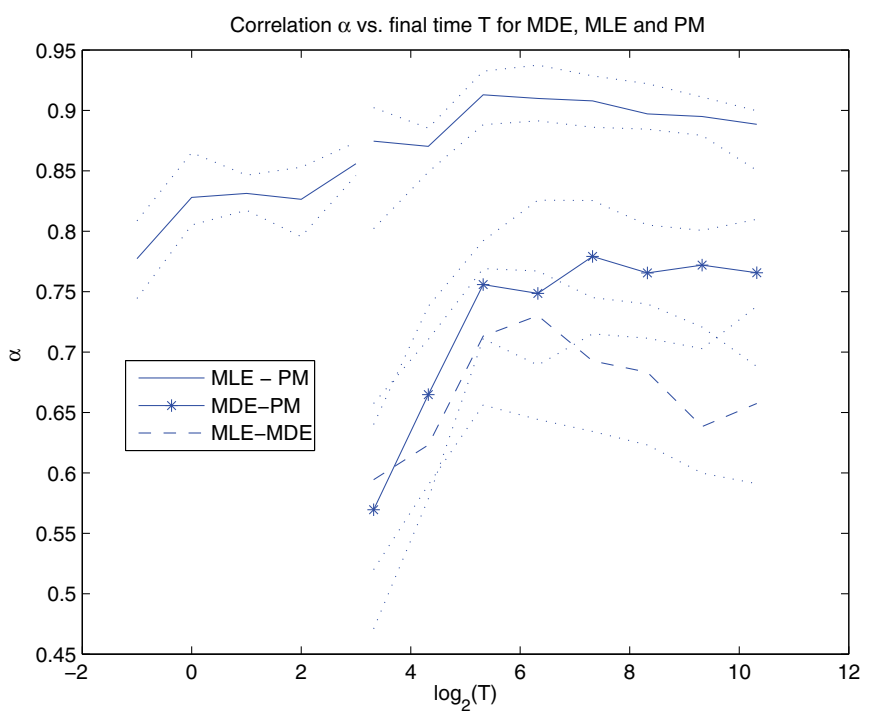

FIG. 4.6. Correlations of drift parameter deviations for MDE, PME, and MLE. The dotted lines indicate $33 \%$ quantile bands.

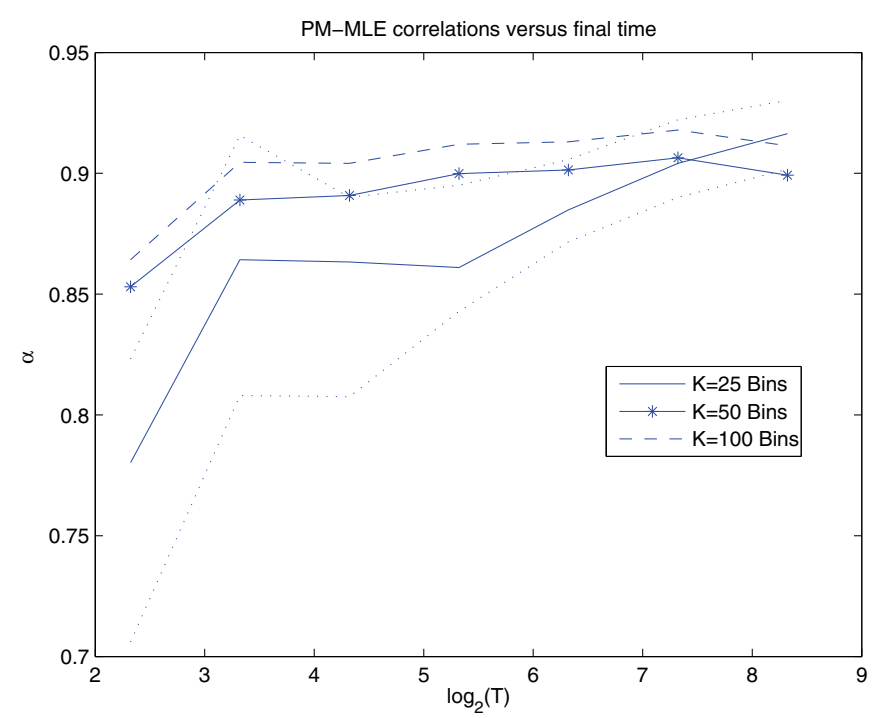

FIG. 4.7. Correlations of drift parameter deviations for MLE and PME. The dotted lines indicate $33 \%$ quantile bands.

It is now appropriate to study correlations as a measure of independence, so we consider the deviations of the three estimators of $\theta_{3}$ from their respective means as a function of final time. Plotting their averaged correlations over at least $N_{a v}=1000$ realizations, each as a function of final time $T$, yields the plot in Figure 4.6. It seems that the maximal obtainable correlation coefficient is around 0.9 for the MLE-PME pair. As would be expected from the analytical link of these estimators, a decline of correlation is observed as the final time $T$ is decreased.

Consulting Figure 4.7, it can be seen that the number of bins has only a small

Copyright $\odot$ by SIAM. Unauthorized reproduction of this article is prohibited. 


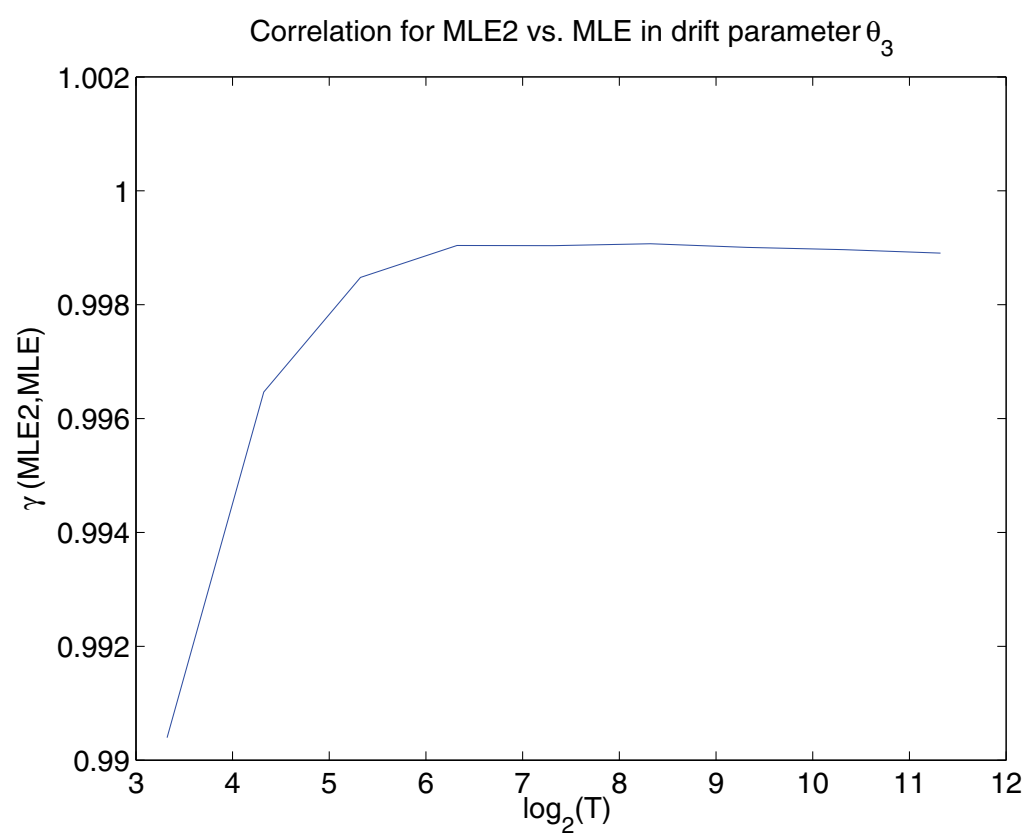

FIG. 4.8. Correlations of drift parameter deviations for $\tilde{A}$ versus MLE.

influence on the observed correlation of the correlation between MLEs and PMEs. We view this as an indication that other smoothing methods arriving at $\hat{\rho}$ would not yield significantly lower correlations.

4.3. Influence of boundary conditions at finite $\boldsymbol{T}$. The approximation of ignoring boundary terms in going from (1.14) to (1.15) is good in the limit of large final times, as was shown in Theorem 2.5. In this section, we will briefly sketch the influence of ignoring these boundary terms for finite, and even small, final times. To do this most easily, we introduce a variant of the maximum likelihood estimator (abbreviated MLE2) obtained by minimizing the following objective function:

$$
\mathcal{I}_{T}^{(2)}[\theta]=\sum_{j=0}^{N}\left(\left|b\left(X_{j}, \theta\right)\right|^{2} \Delta t+\sigma^{2} b^{\prime}\left(X_{j}, \theta\right) \Delta t\right) .
$$

Note that this is similar to a discretization of (2.22), but after having performed a partial integration in the spirit of (2.24) to remove the stochastic integral and neglecting the boundary terms arising from the evaluation of the resulting Stratonovich integral (whereas the MLE would have been attained by discretizing straight away, not performing any partial integrations). It should be compared with $\mathcal{I}_{T}[\theta]$ in (4.6).

In fact, the deviation of the correlation between MLE2 and MLE from 1 should indicate the influence of the initial-condition (and final value) related term on the parameter estimates. Using a similar experimental setup (with $\Delta t=0.0002$ this time), we compute the correlation of the MLE2 and the MLE which results in Figure 4.8.

The remarkably high degree of correlation indicates that the first term, which is of order $\mathcal{O}\left(\frac{1}{T}\right)$, is of little influence for the final times considered in this plot. It does, however, decline for small final times and the onset of this decline around $T=10$ is compatible with the decline of correlation observed in Figure 4.6. 
5. Conclusions and future work. By analyzing different procedures to regularize the likelihood function for the drift of a diffusion, we have highlighted some links between the maximum likelihood principle, used widely in the statistical literature, and the practitioners' estimator based on fitting the logarithm of the empirical measure to the drift. These links have been further substantiated through selected numerical examples. In the special case of gradient diffusions these estimators are even more closely linked, as their deviations from the mean value satisfy the same statistics to leading order.

At first glance the minimum distance estimator seems to be close to the nonparametric approach, but our analysis shows that the link between the parametric approach and the nonparametric approach is far closer.

This paper leaves open many avenues of further enquiry:

- Our work has been exclusively concerned with reversible problems with equilibrium distribution $e^{-\beta V(q)}$, or nonreversible problems with the equilibrium distribution of the Boltzmann-Gibbs form $e^{-\beta H(q, p)}$, with $H(q, p)=\frac{1}{2}|p|^{2}+V(q)$ (separable and quadratic in the momenta). This is natural for examples arising in molecular dynamics. It would also be interesting to perform estimation for processes involving colored noise such as

$$
\ddot{Q}_{t}+\nabla V\left(Q_{t}\right)=B \dot{R}_{t}
$$

where $R_{t}$ is a suitable $m$-dimensional Ornstein-Uhlenbeck process involving $\dot{Q}_{t}$ to satisfy fluctuation dissipation. The process $\left(Q_{t}, \dot{Q}_{t}, R_{t}\right)$ then has marginal measure, after integrating out $R$, of Boltzmann-Gibbs form.

- For problems arising in, e.g., the atmospheric sciences [13], more complex distributions will be required. A characterization of the class of stochastic processes for which the link between the parametric approach and the nonparametric approach can be established would be desirable.

- The option of regularizing the likelihood functional (1.11) by including a higher order differential operator to ensure coercivity has been highlighted. This will be pursued for the one-dimensional case in [19] in the framework of Bayesian nonparametric drift estimation.

- Our results rely heavily on the fact that the diffusion coefficient is assumed known. While it is statistical folklore that drift estimation is considerably harder than diffusion estimation (see, e.g., [23], [12]), in that the quadratic variation in principle reveals the diffusion coefficient, it is common practical experience with real data that diffusion estimation is the harder problem. This is because the data is often incompatible with a diffusion, or with the desired diffusion, at small time-scales; see, e.g., [21] and [3]. To overcome this, practitioners often use time-correlation information, or other information concerning $\mathcal{O}(1)$ time-scales, to estimate the diffusion coefficient; see [8], [18], and [26], for example. Furthermore, multiplicative noise models are often appropriate. See [9] and [13], for example, in the context of molecular dynamics and the atmospheric sciences, respectively, and see also the overview given in [20]. A systematic nonparametric approach to the problem of diffusion matrix estimation in multiple dimensions and for $\mathcal{O}(1)$ spaced data would be very desirable. See [25] for an overview of parametric diffusion estimation in this context. 


\section{Appendix.}

\subsection{Lemma on stochastic integral averages.}

LEMMA 6.1. Let the continuous time stochastic process $X_{t}$ be given by either (1.1) satisfying Assumption 1 or (2.10) satisfying Assumption 2. Also, let $f: \mathbb{R}^{d} \rightarrow \mathbb{R}$ be measurable and polynomially bounded. Then

$$
\lim _{t \rightarrow \infty} \frac{1}{t} \int_{0}^{t}\left\langle f\left(X_{s}\right), d W_{s}\right\rangle=0 \quad \text { a.s. }
$$

We follow the proof given in [17].

Proof. Define the continuous time martingale

$$
M_{t}=\frac{1}{t} \int_{0}^{t}\left\langle f\left(X_{s}\right), d W_{s}\right\rangle
$$

for $t \in(0, \infty)$. Let $\epsilon>0$. For the increasing sequence of times $t_{i}=2^{i-1}, i \in \mathbb{N}$, let the probabilities $p_{i}$ be defined as

$$
p_{i}=\mathbb{P}\left(\sup _{s \in\left[t_{i}, t_{i+1}\right]}\left|M_{s}\right|>\epsilon\right) .
$$

We can bound $p_{i}$ above using the Chebyshev inequality

$$
p_{i} \leq \frac{1}{\epsilon^{2}} \mathbb{E}\left[\sup _{s \in\left[t_{i}, t_{i+1}\right]}\left(\left|M_{s}\right|^{2}\right)\right] .
$$

Now, $|\cdot|^{2}$ is convex and nonnegative and so $\left|M_{s}\right|^{2}$ is a nonnegative submartingale, whence Doob's martingale inequality yields

$$
\begin{aligned}
p_{i} & \leq \frac{C}{\epsilon^{2}} \sup _{s \in\left[t_{i}, t_{i+1}\right]} \mathbb{E}\left[\left|M_{s}\right|^{2}\right] \\
& =\frac{C}{\epsilon^{2}} \sup _{t \in\left[t_{i}, t_{i+1}\right]} \frac{1}{t^{2}} \mathbb{E}\left[\int_{0}^{t}\left|f\left(X_{s}\right)\right|^{2} d s\right]
\end{aligned}
$$

for some constant $C>0$, where we have used the Itô isometry. Since $f$ is polynomially bounded by hypothesis, we can use this bound,

$$
p_{i} \leq \frac{C}{\epsilon^{2}} \sup _{t \in\left[t_{i}, t_{i+1}\right]} \frac{1}{t^{2}} \mathbb{E}\left[\int_{0}^{t} 1+\left|X_{s}\right|^{2 p} d s\right]=\frac{C}{\epsilon^{2}} \sup _{t \in\left[t_{i}, t_{i+1}\right]} \frac{1}{t^{2}} \int_{0}^{t} 1+\mathbb{E}\left[\left|X_{s}\right|^{2 p}\right] d s
$$

for some $p \in \mathbb{N}$ and possibly using a larger constant $C>0$.

For the gradient case, Theorem 5.3 from [16] ensures geometric ergodicity, i.e.,

$$
\left|\mathbb{E} g\left(X_{t}\right)-\int_{\mathbb{R}^{d}} g(a) \rho_{0}(a) d a\right| \leq \kappa\left[1+\left(V\left(X_{0}\right)-\min V\right)^{l}\right] e^{-\lambda t}
$$

for some $\lambda(l), \kappa(l)>0$ and all measurable $g$ such that $|g|<1+(V-\min V)^{l}$. Choosing $l$ large enough so that $|x|^{2 p} \leq V(x)^{l}$ for all $x$ such that $|x|>R$ for some $R>0$, this shows that the integrand is $\mathcal{O}(1)$, i.e., that there exists a constant $C>0$ such that for all times $s \in[0, \infty)$,

$$
\mathbb{E}\left[\left|X_{s}\right|^{2 p}\right]<C
$$

Copyright $@$ by SIAM. Unauthorized reproduction of this article is prohibited. 
For the reversible case, geometric ergodicity is part of Assumption 2 so that (6.1) follows.

Thus, for all $t_{i}>t^{*}$ (hence $i>i^{*}$ ) for some $t^{*}>0$ we have

$$
\begin{aligned}
p_{i} & \leq \sup _{t \in\left[t_{i}, t_{i+1}\right]} \frac{1}{t} \frac{C}{\epsilon^{2}} \quad \forall i \geq i^{*} \\
& \leq \frac{C}{\epsilon^{2}} \frac{1}{t_{i}} \quad \forall i>i^{*}
\end{aligned}
$$

again for a different constant $C$. From the summability $\sum_{i=i^{*}}^{\infty} p_{i}<\infty$ it follows by a Borel-Cantelli lemma that

$$
\mathbb{P}\left(\sup _{s \in\left[t_{i}, t_{i+1}\right]}\left|M_{s}\right|>\epsilon \quad \text { i.o. }\right)=0 .
$$

So that we have $\lim \sup _{s \rightarrow \infty}\left|M_{s}\right| \leq \epsilon$ a.s. Finally, use $\epsilon=\frac{1}{m}, m \in \mathbb{N}$, and let $m \rightarrow \infty$ to obtain the result.

6.2. Unboundedness from below of Brownian bridge functional. Let us consider the random functional

$$
\mathcal{I}_{B}[b]=\int_{0}^{1} b^{2}(x) w(x)+b^{\prime}(x) w(x) d x,
$$

where $b(\cdot) \in H^{1}(0,1)$ and $w(x)$ is a standard Brownian bridge. We claim that this functional is not bounded below and state this as a theorem.

TheOrem 6.2. There a.s. exists a sequence $b^{(n)}(\cdot) \in H^{1}(0,1)$ such that

$$
\lim _{n \rightarrow \infty} \mathcal{I}_{B}\left[b^{(n)}\right]=-\infty \quad \text { a.s. }
$$

Proof. For the Brownian bridge we have the representation

$$
w(x)=\sum_{i=1}^{\infty} \frac{\sin (i \pi x)}{i} \xi_{i},
$$

where the $\left\{\xi_{i}\right\}_{i=1}^{\infty}$ are a sequence of independent and identically distributed normal $\mathcal{N}(0,1)$ random variables. This series converges in $L^{2}\left(\Omega ; L^{2}((0,1), \mathbb{R})\right)$ and a.s. in $C([0,1], \mathbb{R}) ;$ see $[10]$.

Now consider the following sequence of functions $b^{(n)}$ :

$$
b^{(n)}(x)=\sum_{i=1}^{n} \frac{\xi_{i}}{i} \cos (i \pi x) .
$$

We think of a fixed realization $\omega \in \Omega$ of (6.3) for the time being and note that $\{w(x): x \in[0,1]\}$ is a.s. bounded in $L^{\infty}((0,1), \mathbb{R})$, so if there exists a $C>0$ (which may depend on $\left.\left\{\xi_{i}\right\}_{i=0}^{\infty}\right)$ such that

$$
\left\|b^{(n)}\right\|_{L^{2}}<C \quad \forall n \in \mathbb{N},
$$

the first integral in (6.2) will stay finite. By Parseval's identity, it is clear that for the sequence of functionals (6.4) this will be the case if the coefficients $\frac{\xi_{i}}{i}$ are square summable. 
Computing the second summand in (6.2) is straightforward since the series terminates due to orthogonality:

$$
\int_{0}^{1}\left(\sum_{i=1}^{\infty} \frac{\sin (i \pi x)}{i} \xi_{i}\right) \cdot\left(\sum_{j=1}^{n} \frac{\xi_{j}}{j} \cos (j \pi x)\right)^{\prime} d x=-\frac{\pi}{2} \sum_{j=1}^{n} \frac{\xi_{j}^{2}}{j} .
$$

It can now be seen that (6.2) is unbounded from below if the following two conditions are fulfilled:

$$
\begin{aligned}
& \lim _{n \rightarrow \infty} \sum_{j=1}^{n} \frac{1}{j} \xi_{j}^{2}=\infty, \\
& \lim _{n \rightarrow \infty} \sum_{j=1}^{n} \frac{1}{j^{2}} \xi_{j}^{2}<\infty .
\end{aligned}
$$

We finally allow $\omega$ to vary and seek to establish that the conditions (6.6) and (6.7) are a.s. fulfilled. To do this, first note that the random variables being summed are independent. Thus, by the Kolmogorov 0-1 law the probability for convergence is either 0 or 1 . We proceed by applying Kolmogorov's three-series theorem (Theorem 12.5 in [27]) to each of the two sequences to establish (6.6) and (6.7).

We start by treating (6.6). Denote by $\left.X_{j}\right|^{K}$ the truncation of the random variable for some $K>0$ in the following sense:

$$
\left.X_{j}\right|^{K}(\omega)= \begin{cases}X_{j}(\omega) & \text { if }\left|X_{j}(\omega)\right| \leq K \\ 0 & \text { if }\left|X_{j}(\omega)\right|>K\end{cases}
$$

To abbreviate notation, define the following two sequences of random variables:

$$
\begin{aligned}
X_{j} & =\frac{1}{j} \xi_{j}^{2}, \\
Y_{j} & =\frac{1}{j^{2}} \xi_{j}^{2} .
\end{aligned}
$$

Now consider the summability of expected values for the sequence $X_{j}$ : since $\xi_{j}^{2}$ follows a $\chi$-squared distribution with one degree of freedom, its expected value is one. For the truncated variable $\left.X_{j}\right|^{K}$, for any $K>0$, there will be some $j^{*}$ so that for all $j \geq j^{*}$ we have that

$$
\mathbb{E}\left(\left.X_{j}\right|^{K}\right)=\mathbb{E}\left[\frac{1}{j}\left(\left.\xi^{2}\right|^{j K}\right)\right]>\frac{1}{2 j}
$$

Therefore, the expected value summation fails as follows:

$$
\begin{aligned}
\sum_{j=1}^{\infty} \mathbb{E}\left(\left.X_{j}\right|^{K}\right) & =\sum_{j=1}^{\infty} \frac{1}{j} \mathbb{E}\left(\left.\xi^{2}\right|^{j K}\right) \\
& \geq \sum_{j=j^{*}}^{\infty} \frac{1}{2 j}=\infty .
\end{aligned}
$$

Therefore, the series $\sum_{j=1}^{\infty} X_{j}$ diverges to infinity a.s., and thus (6.6) is established. 
Now let us establish (6.7) using the three-series theorem. First check the summability of the expected values:

$$
\sum_{j=1}^{\infty} \mathbb{E}\left(\left.Y_{j}\right|^{K}\right) \leq \sum_{j=1}^{\infty} \mathbb{E} Y_{j}=\sum_{j=1}^{\infty} \frac{1}{j^{2}}<\infty .
$$

Now let us establish the summability of the variances:

$$
\begin{aligned}
\sum_{j=1}^{\infty} \operatorname{Var}\left(\left.Y_{n}\right|^{K}\right) & \leq \sum_{j=1}^{\infty} \operatorname{Var} Y_{n} \\
& =\sum_{j=1}^{\infty} \frac{1}{j^{4}} \operatorname{Var}_{j}^{2} \\
& =2 \sum_{j=1}^{\infty} \frac{1}{j^{4}}<\infty
\end{aligned}
$$

where we used that $\xi_{j}^{2}$ follows a $\chi$-squared distribution with one degree of freedom and hence has variance $\operatorname{Var} \xi_{j}^{2}=2$. Finally, to establish the summability of the tail probabilities we use the following argument for any $K>0$ :

$$
\begin{aligned}
\sum_{j=1}^{\infty} P\left(\left|Y_{j}\right|>K\right) & \leq \sum_{j=1}^{\infty} \frac{1}{K} \mathbb{E}\left|Y_{j}\right| \\
& \leq \frac{1}{K} \sum_{j=1}^{\infty} \frac{1}{j^{2}}<\infty,
\end{aligned}
$$

where we have used the Markov inequality and the previous calculation of the expected value of $Y_{j}=\left|Y_{j}\right|$.

To put everything together, let us reconsider the functional $I[b]$ :

$$
\begin{aligned}
I\left[b^{(n)}\right] & =\int_{0}^{1}\left(b^{(n)}\right)^{2}(x) w(x)+\left(b^{(n)}\right)^{\prime}(x) w(x) d x \\
& \leq\left(\sup _{x \in[0,1]} w(x)\right) \int_{0}^{1}\left(b^{(n)}\right)^{2}(x) d x-\frac{\pi}{2} \sum_{j=1}^{n} \frac{1}{j} \xi_{j}^{2} \\
& \leq\left(\sup _{x \in[0,1]} w(x)\right) \frac{1}{2} \sum_{j=1}^{n} X_{j}-\frac{\pi}{2} \sum_{j=1}^{n} Y_{j} .
\end{aligned}
$$

Now use the a.s. true convergence and divergence statements (6.6) and (6.7) to conclude that

$$
\lim _{n \rightarrow \infty} I\left[b^{(n)}\right]=-\infty \quad \text { a.s. }
$$

\section{REFERENCES}

[1] F. M. BANDi AND P. C. B. Phillips, Fully nonparametric estimation of scalar diffusion models, Econometrica, 71 (2003), pp. 241-283.

[2] F. Comte, V. Genon-Catalot, and Y. Rozenholc, Penalized nonparametric mean square estimation of the coefficients of diffusion processes, Bernoulli, 13 (2007), pp. 514-543.

Copyright $@$ by SIAM. Unauthorized reproduction of this article is prohibited. 
[3] D. T. Crommelin and E. Vanden-Eijnden, Reconstruction of diffusions using spectral data from time-series, Comm. Math. Sci., 4 (2006), pp. 651-668.

[4] R. Durrett, Stochastic Calculus - a Practical Introduction, CRC Press, London, 1996.

[5] L. C. Evans, Partial Differential Equations, AMS, Providence, RI, 1998.

[6] C. W. Gardiner, Handbook of Stochastic Methods, Springer, Berlin, 1985.

[7] E. Gobet, M. Hoffmann, And M. Reiss, Nonparametric estimation of scalar diffusions based on low frequency data, Ann. Statist., 32 (2004), pp. 2223-2253.

[8] H. Grubmüller AND P. TAVAN, Molecular dynamics of conformational substates for a simplified protein model, J. Chem. Phys., 101 (1994), pp. 5047-5057.

[9] G. Hummer, Position-dependent diffusion coefficients and free energies from Bayesian analysis of equilibrium and replica molecular dynamics simulations, New J. Phys., 7 (2005), paper 34.

[10] J. P. Kahane, Some Random Series of Functions, Cambridge University Press, Cambridge, UK, 1985.

[11] O. Kallenberg, Foundations of Modern Probability, 2nd ed., Springer-Verlag, New York, 2002.

[12] Y. A. Kutoyants, Statistical Inference for Ergodic Diffusion Processes, Springer-Verlag, London, 2004.

[13] A. J. Majda, I. Timofeyev, and E. Vanden-Eijnden, A mathematical framework for stochastic climate models, Comm. Pure Appl. Math., 54 (2001), pp. 891-974.

[14] X. MAO, Stochastic Differential Equations and Applications, 2nd ed., Horwood, Chichester, UK, 2008.

[15] M. B. Marcus and J. Rosen, Markov Processes, Gaussian Processes, and Local Times, Cambridge University Press, Cambridge, UK, 2006.

[16] J. Mattingly, A. M. Stuart, and D. J. Higham, Ergodicity for SDEs and approximations: Locally Lipschitz vector fields and degenerate noise, Stochastic Process. Appl., 101 (2002), pp. $185-232$.

[17] J. C. Mattingly, A. M. Stuart, and M. V. Tretyakov, Convergence of Numerical Time-Averaging and Stationary Measures via Poisson Equations, http://arxiv.org/ abs/0908.4450 (2009).

[18] W. Nadler, A. T. Brünger, K. Schulten, and M. Karplus, Molecular and stochastic dynamics of proteins, Proc. Nat. Acad. Sci. USA, 84 (1987), pp. 7933-7937.

[19] O. Papaspiliopoulos, Y. Pokern, G. O. Roberts, and A. M. Stuart, Nonparametric Bayesian drift estimation for one-dimensional SDEs, submitted.

[20] G. A. Pavliotis, Y. Pokern, and A. M. Stuart, Parameter estimation for multiscale diffusions: An overview, in Statistical Methods for Stochastic Differential Equations, Semstat 2007 Proceedings, M. Kessler, A. Lindner, and M. Sørensen, eds., Chapman \& Hall, London, to appear.

[21] G. Pavliotis and A. M. Stuart, Parameter estimation for multiscale diffusions, J. Stat. Phys., 127 (2007), pp. 741-781.

[22] N. Privault and A. RÉveillac, Superefficient drift estimation on the Wiener space, C. R. Math. Acad. Sci. Paris, 343 (2006), pp. 607-612.

[23] B. L. S. Prakasa RaO, Statistical Inference for Diffusion Type Processes, Arnold Publishers, London, 1999.

[24] H. Risken, The Fokker-Planck Equation, 2nd ed., Springer-Verlag, Berlin, 1989.

[25] G. O. RoBerTs, Exact Simulation and Inference for Diffusions, presentation and lecture notes, Statistical Methods for Stochastic Differential Equations (SemStat), Cartagena, Spain, 2007.

[26] J. E. Straub, M. Borkovec, And B. J. Berne, Calculation of dynamic friction on intramolecular degrees of freedom, J. Phys. Chem., 91 (1987), pp. 4995-4998.

[27] D. Williams, Probability with Martingales, Cambridge University Press, Cambridge, UK, 1991.

Copyright (c) by SIAM. Unauthorized reproduction of this article is prohibited. 